\title{
Topology on new sequence spaces defined with wavelet leaders
}

\author{
F. Bastin, C. Esser* ${ }^{*}$ L. Simons \\ Institute of Mathematics B37, University of Liège, Quartier Polytech 1, Allée de la Découverte 12, \\ B-4000 Liège, Belgium
}

\section{A R T I C L E I N F O}

Article history:

Received 12 January 2015

Available online 27 May 2015

Submitted by E. Saksman

\section{Keywords:}

Sequence spaces

Topology on vector spaces

Wavelets

\begin{abstract}
A B S T R A C T
Using wavelet leaders instead of wavelet coefficients, new sequence spaces of type $\mathcal{S}^{\nu}$ are defined and endowed with a natural topology. Some classical topological properties are then studied; in particular, a generic result about the asymptotic repartition of the wavelet leaders of a sequence in $\mathcal{L}^{\nu}$ is obtained. Eventually, comparisons and links with Oscillation spaces are also presented as well as with $\mathcal{S}^{\nu}$ spaces.
\end{abstract}

(ㄷ) 2015 Elsevier Inc. All rights reserved.

\section{Introduction}

The study of the regularity of a signal by means of its wavelet coefficients is now a widely used tool. Mathematically, it involves the use of sequence spaces which are supposed to constitute an appropriate setting to handle the information. In order to study the regularity of a signal via the distribution of its wavelet coefficients, $\mathcal{S}^{\nu}$ spaces have been introduced and it has been shown that they contain more information than the classical Besov spaces (see [13]). Nevertheless, the use of these $\mathcal{S}^{\nu}$ spaces presents some weaknesses and then, new spaces of the same type have recently been introduced using wavelet leaders instead of wavelet coefficients (see [8]). These spaces are denoted by $\mathcal{L}^{\nu}$.

Before giving more details about the introduction and the definition of $\mathcal{L}^{\nu}$ spaces, let us be more precise about the notion of regularity. Let $x_{0} \in \mathbb{R}$ and $\alpha \geq 0$. A locally bounded function $f: \mathbb{R} \rightarrow \mathbb{R}$ belongs to the Hölder space $C^{\alpha}\left(x_{0}\right)$ if there exist a constant $C>0$ and a polynomial $P$ of degree strictly less than $\alpha$ such that

$$
|f(x)-P(x)| \leq C\left|x-x_{0}\right|^{\alpha}
$$

for all $x$ in a neighbourhood of $x_{0}$. The Hölder exponent of $f$ at $x_{0}$ is defined by

\footnotetext{
* Corresponding author.

E-mail addresses: F.Bastin@ulg.ac.be (F. Bastin), Celine.Esser@ulg.ac.be (C. Esser), L.Simons@ulg.ac.be (L. Simons).
} 


$$
h_{f}\left(x_{0}\right):=\sup \left\{\alpha \geq 0: f \in C^{\alpha}\left(x_{0}\right)\right\}
$$

and the multifractal spectrum of $f$ is the function $d_{f}$ defined by

$$
d_{f}(h):=\operatorname{dim}_{\mathcal{H}}\left\{x \in \mathbb{R}: h_{f}(x)=h\right\}, \quad h \in[0,+\infty]
$$

(where $\operatorname{dim}_{\mathcal{H}}$ denotes the Hausdorff dimension). This function gives a geometrical idea about the distribution of the singularities of $f$. For a general signal (i.e. a function obtained from real-life data), it is clearly impossible to estimate $d_{f}$ numerically since it involves the successive determination of several intricate limits. Therefore one tries instead to estimate this spectrum from quantities which are numerically computable. Such a method is called a multifractal formalism.

The Frisch-Parisi conjecture, classically used, gives such an estimation based on a wavelet decomposition and the use of Besov spaces (see [23,12]). Nevertheless, it appeared that this use of Besov spaces is not sufficient to handle all the information concerning the pointwise regularity contained in the distribution of the wavelet coefficients (see [13]). In particular, it can only lead to recover increasing and concave hull of spectra.

In order to get a suitable context to obtain multifractal results in the non-concave case, $\mathcal{S}^{\nu}$ spaces have then been introduced (see [13]). These spaces contain the maximal information that can be derived from the repartition at every scale of the wavelet coefficients of a function. They have been studied in several papers: topological (and specific functional analysis) results were obtained, as well as answers for multifractal formalisms (see $[5,6,4,1,3,2]$ ). An implementation of this formalism has been proposed and tested on several theoretical examples in [18]. However, the $\mathcal{S}^{\nu}$ spaces can only detect increasing part of spectra.

Meanwhile, it appeared that more accurate information concerning the pointwise regularity can be obtained when relying on wavelet leaders, which can be seen as local suprema of wavelet coefficients. Indeed, wavelet leaders give an easier characterization of the pointwise regularity than wavelet coefficients (see for example [16] and references therein). In particular, they allow to obtain information about the inter-scale organization of the wavelet coefficients, without making any a priori probabilistic assumptions on their repartition. In this context, Oscillation spaces have been introduced as a generalization of Besov spaces using wavelet leaders (see [15]) and multifractal results have been obtained (see [14,16]). In particular, Oscillation spaces gives a method which allows to recover increasing and decreasing parts of spectra. Nevertheless, this method is still limited to concave spectra. So, a natural idea was to extend the study of the $\mathcal{S}^{\nu}$ spaces (defined directly using the wavelet coefficients) to the context of wavelet leaders. Those spaces, called $\mathcal{L}^{\nu}$ spaces and introduced in [8], lead to better approximations for non-concave spectra with a decreasing part. Several positive results have been obtained in [8]. Moreover, in [18], the different formalisms (based on Oscillation spaces, $\mathcal{S}^{\nu}$ spaces and $\mathcal{L}^{\nu}$ spaces) have been compared. It appeared that the method based on the $\mathcal{L}^{\nu}$ spaces is more efficient from the theoretical point of view and that in practice, it gives complementary results to those obtained using the formalism based on Oscillation spaces.

In this paper, in order to understand better the structure of the $\mathcal{L}^{\nu}$ spaces, we endow them with a topology. As done in the case of the $\mathcal{S}^{\nu}$ spaces (see $[3,4,6]$ ), one of our purposes is to get applications in multifractal analysis and in particular, to obtain the generic validity of the multifractal formalism based on $\mathcal{L}^{\nu}$ spaces. This would give a theoretical justification to this method. Indeed, as for the other multifractal formalisms, the method based on the $\mathcal{L}^{\nu}$ spaces never holds in complete generality, but it yields an upper bound for the multifractal spectrum of all functions in the space $\mathcal{L}^{\nu}$ (see [8]). This is the best that can be expected: usually, there are no non-trivial minorations for the multifractal spectrum of all functions in the space. Nevertheless, one can hope that for most of the functions in the space, that is to say for a generic subset of the space (in the sense of Baire categories), the inequality becomes an equality.

Let us give some classical notations used in the paper. The set of strictly positive natural numbers is $\mathbb{N}$ and we denote $\mathbb{N}_{0}:=\{0\} \cup \mathbb{N}$. We use the notation $\lambda$ to refer to the dyadic interval 


$$
\lambda=\lambda(j, k):=\left\{x \in \mathbb{R}: 2^{j} x-k \in\left[0,1[\}=\left[\frac{k}{2^{j}}, \frac{k+1}{2^{j}}\left[, \quad j \in \mathbb{N}_{0}, k \in\left\{0, \ldots, 2^{j}-1\right\},\right.\right.\right.\right.
$$

and for all $j \in \mathbb{N}_{0}, \Lambda_{j}$ denotes the set of all dyadic intervals (of $\left[0,1\left[\right.\right.$ ) of length $2^{-j}$. We write \#A for the cardinal of the set $A$.

Let us also recall some useful notions and notations which lead to define $\mathcal{S}^{\nu}$ and $\mathcal{L}^{\nu}$ spaces as introduced in $[13,14,8]$. Since we are interested in local properties of functions, we can suppose from now on that the functions we consider are 1-periodic. We take a mother wavelet $\psi$ in the Schwartz class (as done in [19]) and we write

$$
\psi_{j, k}(\cdot):=\sum_{l \in \mathbb{Z}} \psi\left(2^{j}(\cdot-l)-k\right), \quad j \in \mathbb{N}_{0}, k \in\left\{0, \ldots, 2^{j}-1\right\}
$$

The 1-periodic functions $2^{j / 2} \psi_{j, k}, j \in \mathbb{N}_{0}, k \in\left\{0, \ldots, 2^{j}-1\right\}$, together with the constant function 1 form an orthonormal basis of the space of the 1-periodic functions of $L^{2}([0,1])$ (see $[9,20,22]$ for more details). The wavelet coefficients of such a function $f$ are defined by

$$
c_{j, k}:=2^{j} \int_{0}^{1} f(x) \psi_{j, k}(x) d x, \quad j \in \mathbb{N}_{0}, k \in\left\{0, \ldots, 2^{j}-1\right\}
$$

(where we have used an $L^{\infty}$-normalization to simplify notations and formulas). We will also use the notations $\psi_{\lambda}$ and $c_{\lambda}$ instead of $\psi_{j, k}$ and $c_{j, k}$ respectively.

The wavelet leaders of a signal $f \in L^{2}([0,1])$ are defined by

$$
d_{j, k}=d_{\lambda}:=\sup _{\lambda^{\prime} \subset \lambda}\left|c_{\lambda^{\prime}}\right|, \quad \lambda \in \Lambda_{j}, j \in \mathbb{N}_{0} .
$$

With this definition, it may happen that $d_{\lambda}=+\infty$. To ensure that this is not the case, it is of course sufficient to assume that the signal $f$ belongs to $L^{\infty}([0,1])$ as presented in [14]. Let us remark that in the context of pointwise Hölder regularity, the wavelets leaders are usually defined as follows:

$$
d_{\lambda}^{*}:=\sup _{\lambda^{\prime} \subset 3 \lambda}\left|c_{\lambda^{\prime}}\right|, \quad \lambda \in \Lambda_{j}, j \in \mathbb{N}_{0},
$$

where $3 \lambda$ denotes the cube of the same center as $\lambda$ but three times larger. This " $3 \lambda$ " is motivated by the fact that the pointwise regularity is characterized in terms of these "selected coefficients". As shown in [8], this choice is no longer justified in our case since both definitions of wavelet leaders give the same spaces $\mathcal{L}^{\nu}$.

For all $C>0, \alpha \in \mathbb{R}$ and $j \in \mathbb{N}_{0}$, we write

$$
\widetilde{E_{j}}(C, \alpha)(f):=\left\{k \in\left\{0, \ldots, 2^{j}-1\right\}: d_{j, k} \geq C 2^{-\alpha j}\right\}=\left\{\lambda \in \Lambda_{j}: d_{\lambda} \geq C 2^{-\alpha j}\right\} .
$$

Following [8], the (increasing) wavelet leaders profile of $f \in L^{\infty}([0,1])$ is the function $\widetilde{\nu}_{f}$ defined by

$$
\widetilde{\nu}_{f}(\alpha):=\lim _{\varepsilon \rightarrow 0^{+}}\left(\limsup _{j \rightarrow+\infty}\left(\frac{\log \left(\# \widetilde{E_{j}}(1, \alpha+\varepsilon)(f)\right)}{\log \left(2^{j}\right)}\right)\right), \quad \alpha \geq 0 .
$$

Then there is $\alpha_{0} \geq 0$ such that $\widetilde{\nu}_{f}(\alpha)=-\infty$ for every $\alpha<\alpha_{0}$ and we extend this function by setting $\widetilde{\nu}_{f}(\alpha)=$ $-\infty$ for $\alpha<0$ (similarly to the classical wavelet profile of $f$ in [13]). This definition formalizes the idea that at large scales $j$, there are about $2^{\widetilde{\nu}_{f}(\alpha) j}$ wavelet leaders larger than $2^{-\alpha j}$ (with the convention $2^{-\infty}:=0$ ). By construction, the function $\widetilde{\nu}_{f}$ is non-decreasing, right-continuous and with values in $\{-\infty\} \cup[0,1]$. Moreover, $\widetilde{\nu}_{f}$ is independent of the chosen wavelet basis (see [8]). 
In the context of functions, an admissible profile is a function $\nu$ defined on $\mathbb{R}$, non-decreasing, rightcontinuous, with values in $\{-\infty\} \cup[0,1]$ and for which

$$
0 \leq \alpha_{\min }:=\inf \{\alpha \in \mathbb{R}: \nu(\alpha) \geq 0\}
$$

In particular, any wavelet leaders profile is admissible. Given an admissible profile $\nu$, the space $\mathcal{L}^{\nu}$ is defined as the set of functions $f$ such that

$$
\widetilde{\nu}_{f}(\alpha) \leq \nu(\alpha) \quad \forall \alpha \in \mathbb{R}
$$

Since the wavelet leaders profile is independent of the chosen wavelet basis, the same holds for the space $\mathcal{L}^{\nu}$. Therefore, as in the case of $\mathcal{S}^{\nu}$ spaces (see [5]), we can consider $\mathcal{L}^{\nu}$ as a sequence space (and no more as a function space), independently of the context they come from. That is the point of view that we adopt here.

The paper is organized as follows. In Section 2, we recall basic definitions and results about $\mathcal{S}^{\nu}$ spaces. In Section 3, we reintroduce $\mathcal{L}^{\nu}$ spaces as sequence spaces and endow those spaces with a natural topology. Some properties of this topological vector space are also studied. In Section 4, we present the generic form of the wavelet leaders profile of a sequence in $\mathcal{L}^{\nu}$. In Section 5 , we look at the existing inclusions between spaces of type $\mathcal{S}^{\nu}$ and spaces of type $\mathcal{L}^{\nu}$. Finally, as done for $\mathcal{S}^{\nu}$ spaces and Besov spaces, we compare $\mathcal{L}^{\nu}$ spaces and Oscillation spaces in Section 6.

Let us end by mentioning that in [8], a more general definition of $\mathcal{L}^{\nu}$ spaces is considered: a decreasing wavelet leaders profile is also defined. Nevertheless, if the admissible profile has a decreasing part, the corresponding $\mathcal{L}^{\nu}$ space is not a vector space. This is the reason why, in this paper, we work only with increasing admissible profiles.

\section{Some definitions and known results about $\mathcal{S}^{\nu}$ spaces}

Let us recall some definitions and some basic topological results obtained for $\mathcal{S}^{\nu}$ spaces (see [5] for details and $[4,1,3,2]$ for more results).

\subsection{Definitions}

Let us denote $\Lambda:=\bigcup_{j \in \mathbb{N}_{0}}\{j\} \times\left\{0, \ldots, 2^{j}-1\right\}$ and define $\Omega:=\mathbb{C}^{\Lambda}$. In the context of sequence spaces, an admissible profile is a non-decreasing right-continuous function of a real variable, with values in $\{-\infty\} \cup[0,1]$ such that

$$
\alpha_{\min }:=\inf \{\alpha \in \mathbb{R}: \nu(\alpha) \geq 0\} \in \mathbb{R}
$$

Let us mention that one can take $\alpha_{\min }<0$ because we work with sequences in $\Omega$; let us remark that in the context of locally bounded functions, we always have $\alpha_{\min } \geq 0$.

Following [5], the wavelet profile of a sequence $\vec{c} \in \Omega$ is the function $\nu_{\vec{c}}$ defined by

$$
\nu_{\vec{c}}(\alpha):=\lim _{\varepsilon \rightarrow 0^{+}}\left(\limsup _{j \rightarrow+\infty}\left(\frac{\log \left(\# E_{j}(1, \alpha+\varepsilon)(\vec{c})\right)}{\log \left(2^{j}\right)}\right)\right), \quad \alpha \in \mathbb{R}
$$

where

$$
E_{j}(C, \alpha)(\vec{c}):=\left\{k \in\left\{0, \ldots, 2^{j}-1\right\}:\left|c_{j, k}\right| \geq C 2^{-\alpha j}\right\}
$$


for $j \in \mathbb{N}_{0}, C>0$ and $\alpha \in \mathbb{R}$. Given an admissible profile $\nu$, a sequence $\vec{c}$ belongs to $\mathcal{S}^{\nu}$ if

$$
\nu_{\vec{c}}(\alpha) \leq \nu(\alpha) \quad \forall \alpha \in \mathbb{R}
$$

Equivalently, $\vec{c}$ belongs to $\mathcal{S}^{\nu}$ if and only if for every $\alpha \in \mathbb{R}, \varepsilon>0$ and $C>0$, there exists $J \in \mathbb{N}_{0}$ such that

$$
\# E_{j}(C, \alpha)(\vec{c}) \leq 2^{(\nu(\alpha)+\varepsilon) j} \quad \forall j \geq J
$$

When $\nu(\alpha)=-\infty$, we use the convention $2^{-\infty j}:=0$ for all $j \in \mathbb{N}_{0}$. Heuristically, a sequence $\vec{c}$ belongs to $\mathcal{S}^{\nu}$ if at each large scale $j$, the number of $k$ such that $\left|c_{j, k}\right| \geq 2^{-\alpha j}$ is of order smaller than $2^{\nu(\alpha) j}$. This space is a vector space (see Section 2 in [5]).

\subsection{Basic results}

In this subsection, we summarize the topological properties of $\mathcal{S}^{\nu}$ established in [5].

There exists a unique metrizable topology that is stronger than the topology of the pointwise convergence (by definition, a sequence $\left(\vec{c}^{(m)}\right)_{m \in \mathbb{N}}$ converges pointwise to $\vec{c}$ if for all $j \in \mathbb{N}_{0}$ and all $k \in\left\{0, \ldots, 2^{j}-1\right\}$, $c_{j, k}^{(m)} \rightarrow c_{j, k}$ in $\mathbb{C}$ if $\left.m \rightarrow+\infty\right)$ and that makes $\mathcal{S}^{\nu}$ a complete topological vector space. This topology is separable, the compact sets have been characterized, and the link with Besov spaces has been obtained. Since the aim of the present paper is to prove similar results using wavelet leaders, let us recall more precise results here below.

Let us first recall the definition of the Besov sequence spaces. They are discrete counterpart of Besov spaces of functions (see [22]). More precisely, for $s \in \mathbb{R}$ and $p>0$, the Besov space $b_{p, \infty}^{s}$ is the set of sequences $\vec{c} \in \Omega$ such that

$$
\|\vec{c}\|_{b_{p, \infty}^{s}}:=\sup _{j \in \mathbb{N}_{0}} 2^{\left(s-\frac{1}{p}\right) j}\left(\sum_{k=0}^{2^{j}-1}\left|c_{j, k}\right|^{p}\right)^{\frac{1}{p}}<+\infty .
$$

The definition is extended to the case $p=\infty$ by setting $b_{\infty, \infty}^{s}=C^{s}$ with

$$
\|\vec{c}\|_{C^{s}}:=\sup _{j \in \mathbb{N}_{0}} \sup _{k \in\left\{0, \ldots, 2^{j}-1\right\}} 2^{s j}\left|c_{j, k}\right| .
$$

This corresponds to the Hölder space of order $s$. If we define the concave conjugate $\eta$ of the admissible profile $\nu$ by

$$
\eta(p):=\inf _{\alpha \geq \alpha_{\min }}(\alpha p-\nu(\alpha)+1), \quad p>0
$$

we get the following embedding of $\mathcal{S}^{\nu}$ spaces into Besov spaces (see Proposition 8.7 in [5]).

Proposition 2.1. If $\left(p_{n}\right)_{n \in \mathbb{N}}$ is a dense sequence of $] 0,+\infty\left[\right.$ and if $\left(\varepsilon_{m}\right)_{m \in \mathbb{N}}$ is a sequence of strictly positive numbers converging to 0 , then

$$
\mathcal{S}^{\nu} \subset \bigcap_{\varepsilon>0} \bigcap_{p>0} b_{p, \infty}^{\frac{\eta(p)}{p}-\varepsilon}=\bigcap_{n \in \mathbb{N}} \bigcap_{m \in \mathbb{N}} b_{p_{n}, \infty}^{\frac{\eta\left(p_{n}\right)}{p_{n}}-\varepsilon_{m}}
$$

and this inclusion becomes an equality if and only if $\nu$ is concave. 
This result justifies the introduction of the $\mathcal{S}^{\nu}$ spaces: the spaces $\bigcap_{\varepsilon>0} \bigcap_{p>0} b_{p, \infty}^{\eta(p) / p-\varepsilon}$ do not contain more information about the multifractal spectra of their elements than their concave hull since for most of the functions in this intersection, the spectrum of singularities is given by a Fenchel-Legendre transform of $\eta$ (see [12]). If $\nu$ is not concave, the space $\mathcal{S}^{\nu}$ gives an additional information and leads to estimation of spectra which are not concave. We refer the reader to $[3,4,7,18]$ for more information about this method.

In order to define a complete metrizable topology on $\mathcal{S}^{\nu}$, auxiliary spaces were introduced. For any $\alpha \in \mathbb{R}$ and any $\beta \in\{-\infty\} \cup[0,+\infty[$, the space $A(\alpha, \beta)$ is defined by

$$
A(\alpha, \beta):=\left\{\vec{c} \in \Omega: \exists C, C^{\prime} \geq 0 \text { such that } \# E_{j}(C, \alpha)(\vec{c}) \leq C^{\prime} 2^{\beta j} \forall j \in \mathbb{N}_{0}\right\} .
$$

This space is endowed with the distance

$$
\delta_{\alpha, \beta}\left(\vec{c}, \vec{c}^{\prime}\right):=\inf \left\{C+C^{\prime}: C, C^{\prime} \geq 0 \text { and } \# E_{j}(C, \alpha)\left(\vec{c}-\vec{c}^{\prime}\right) \leq C^{\prime} 2^{\beta j} \forall j \in \mathbb{N}_{0}\right\}, \quad \vec{c}, \vec{c}^{\prime} \in A(\alpha, \beta) .
$$

Remark that if $\beta=-\infty$, then $\left(A(\alpha,-\infty), \delta_{\alpha,-\infty}\right)$ is the topological normed space $C^{\alpha}$. If $\beta \geq 1$, then $A(\alpha, \beta)=\Omega$. Moreover, in case $\beta>1$, the topology defined by the distance $\delta_{\alpha, \beta}$ is equivalent to the topology of pointwise convergence.

Proposition 2.2. For any sequence $\left(\alpha_{n}\right)_{n \in \mathbb{N}}$ dense in $\mathbb{R}$ and any sequence $\left(\varepsilon_{m}\right)_{m \in \mathbb{N}}$ of strictly positive numbers decreasing to 0 , we have

$$
\mathcal{S}^{\nu}=\bigcap_{m \in \mathbb{N}} \bigcap_{n \in \mathbb{N}} A\left(\alpha_{n}, \nu\left(\alpha_{n}\right)+\varepsilon_{m}\right)
$$

The topology of $\mathcal{S}^{\nu}$ is defined as the projective limit topology, i.e. the coarsest topology that makes each inclusion $\mathcal{S}^{\nu} \subset A\left(\alpha_{n}, \nu\left(\alpha_{n}\right)+\varepsilon_{m}\right)$ continuous. This topology is equivalent to the topology given by the distance

$$
\delta=\sum_{m=1}^{+\infty} \sum_{n=1}^{+\infty} 2^{-(m+n)} \frac{\delta_{m, n}}{1+\delta_{m, n}}
$$

where $\delta_{m, n}$ denotes the distance $\delta_{\alpha_{n}, \nu\left(\alpha_{n}\right)+\varepsilon_{m}}$ (see Section 5 in [5]).

\section{3. $\mathcal{L}^{\nu}$ spaces}

\subsection{Definitions and first properties}

Let us first introduce the definition of the $\mathcal{L}^{\nu}$ spaces in the context of sequence spaces. If $\vec{c} \in \Omega$, we define

$$
d_{\lambda}:=\sup _{\lambda^{\prime} \subset \lambda}\left|c_{\lambda^{\prime}}\right|, \quad \lambda \in \Lambda_{j}, j \in \mathbb{N}_{0} .
$$

With this definition, it may happen that $d_{\lambda}=+\infty$. However, in what follows, we will see that the definition of $\mathcal{L}^{\nu}$ spaces leads to wavelet leaders which are always finite.

Following [8], we consider the definitions below which provide a generalization of the wavelet profile and the $\mathcal{S}^{\nu}$ spaces with wavelet leaders.

Definition 3.1. The wavelet leaders profile of $\vec{c} \in \Omega$ is the function $\widetilde{\nu}_{\vec{c}}$ defined by

$$
\widetilde{\nu}_{\vec{c}}(\alpha):=\lim _{\varepsilon \rightarrow 0^{+}}\left(\limsup _{j \rightarrow+\infty}\left(\frac{\log \left(\# \widetilde{E_{j}}(1, \alpha+\varepsilon)(\vec{c})\right)}{\log \left(2^{j}\right)}\right)\right), \quad \alpha \in \mathbb{R},
$$


where

$$
\widetilde{E_{j}}(C, \alpha)(\vec{c}):=\left\{k \in\left\{0, \ldots, 2^{j}-1\right\}: d_{j, k} \geq C 2^{-\alpha j}\right\}
$$

for $j \in \mathbb{N}_{0}, C>0$ and $\alpha \in \mathbb{R}$.

Definition 3.2. Given an admissible profile $\nu$, the space $\mathcal{L}^{\nu}$ is the set of sequences $\vec{c} \in \Omega$ such that

$$
\widetilde{\nu}_{\vec{c}}(\alpha) \leq \nu(\alpha) \quad \forall \alpha \in \mathbb{R}
$$

Remark 3.3. As already mentioned in the end of the introduction, we do not consider here the case where $\nu$ has a decreasing part (in comparison with the definition of an admissible profile given in [8]). We so ensure that $\mathcal{L}^{\nu}$ is a vector space.

Just as in the case of $\mathcal{S}^{\nu}$ spaces, we get the following description of $\mathcal{L}^{\nu}$ (the proof is a simple adaptation of the proof of Lemma 2.3 in [5]).

Proposition 3.4. Let $\nu$ be an admissible profile. The space $\mathcal{L}^{\nu}$ is a vector space and a sequence $\vec{c} \in \Omega$ belongs to $\mathcal{L}^{\nu}$ if and only if for every $\alpha \in \mathbb{R}, \varepsilon>0$ and $C>0$, there exists $J \in \mathbb{N}_{0}$ such that

$$
\# \widetilde{E_{j}}(C, \alpha)(\vec{c}) \leq 2^{(\nu(\alpha)+\varepsilon) j} \quad \forall j \geq J
$$

Let us remark that if a sequence belongs to $\mathcal{L}^{\nu}$, it is in $C^{0}$ and therefore, its wavelet leaders are finite. Indeed, if $\alpha<\alpha_{\min }$, there is $C>0$ such that

$$
d_{j, k} \leq C 2^{-\alpha j} \quad \forall j \in \mathbb{N}_{0}, k \in\left\{0, \ldots, 2^{j}-1\right\}
$$

In particular, $d_{0,0}=\sup _{j \in \mathbb{N}_{0}, k \in\left\{0, \ldots, 2^{j}-1\right\}}\left|c_{j, k}\right| \leq C$.

Remark 3.5. In [8], the spaces $\mathcal{L}^{\nu}$ are studied as spaces of locally bounded functions. The assumption $\alpha_{\min } \geq 0$ is therefore always satisfied. Let us remark that in the context of sequences, we can also restrict ourselves to this case. Indeed, let us assume that $\alpha_{\min }<0$ and define $\nu^{\dagger}$ as follows:

$$
\nu^{\dagger}(\alpha):= \begin{cases}\nu(\alpha) & \text { if } \alpha \geq 0 \\ -\infty & \text { if } \alpha<0\end{cases}
$$

Then, since $\mathcal{L}^{\nu} \subset C^{0}$, we get

$$
\mathcal{L}^{\nu}=\mathcal{L}^{\nu^{\dagger}}
$$

Therefore, from now on, we will always assume that $\nu$ is an admissible profile with $\alpha_{\text {min }} \geq 0$. Let us already note that in Section 4 we will show that any admissible profile is the wavelet leaders profile of a sequence in $C^{0}$. This justifies the definition of an admissible profile.

\subsection{Auxiliary spaces}

As for the case of the $\mathcal{S}^{\nu}$ spaces, a useful description can also be obtained by the introduction of auxiliary spaces. These new spaces will be used to define a topology on $\mathcal{L}^{\nu}$. 
Definition 3.6. Let $\alpha \in \mathbb{R}$ and $\beta \in\{-\infty\} \cup[0,+\infty[$. A sequence $\vec{c} \in \Omega$ belongs to the auxiliary space $\widetilde{A}(\alpha, \beta)$ if there exist $C, C^{\prime} \geq 0$ such that

$$
\# \widetilde{E_{j}}(C, \alpha)(\vec{c}) \leq C^{\prime} 2^{\beta j} \quad \forall j \in \mathbb{N}_{0}
$$

Let us remark that the auxiliary spaces are vector spaces. Moreover, we have the following result.

Proposition 3.7. For any dense sequence $\left(\alpha_{n}\right)_{n \in \mathbb{N}}$ in $\mathbb{R}$ and any sequence $\left(\varepsilon_{m}\right)_{m \in \mathbb{N}}$ of strictly positive numbers which converges to 0 , we have

$$
\mathcal{L}^{\nu}=\bigcap_{\varepsilon>0} \bigcap_{\alpha \in \mathbb{R}} \widetilde{A}(\alpha, \nu(\alpha)+\varepsilon)=\bigcap_{m \in \mathbb{N}} \bigcap_{n \in \mathbb{N}} \widetilde{A}\left(\alpha_{n}, \nu\left(\alpha_{n}\right)+\varepsilon_{m}\right)
$$

Proof. This proof is a simple adaptation of the proof of Theorem 5.4 in [5] using spaces $\widetilde{A}(\alpha, \beta)$ instead of $A(\alpha, \beta)$.

Let us give some first remarks and properties related to these auxiliary spaces.

\section{Remark 3.8.}

1. If $\beta=-\infty$, then $\widetilde{A}(\alpha, \beta)$ is the set of the sequences $\vec{c} \in \Omega$ satisfying

$$
\sup _{j \in \mathbb{N}_{0}} \sup _{k \in\left\{0, \ldots, 2^{j}-1\right\}} 2^{j \alpha} d_{j, k}<+\infty .
$$

Let us remark that it is the Hölder space $C^{\alpha}$ if $\alpha>0$ and the Hölder space $C^{0}$ if $\alpha \leq 0$. Indeed, if $\alpha>0$, this follows from the fact that if there is $R>0$ such that $\sup _{j, k} 2^{\alpha j}\left|c_{j, k}\right| \leq R$ then $\left|c_{j^{\prime}, k^{\prime}}\right| \leq 2^{-\alpha j^{\prime}} R \leq$ $2^{-\alpha j} R$ for every $j^{\prime} \geq j, k^{\prime} \in\left\{0, \ldots, 2^{j^{\prime}}-1\right\}$. Hence $d_{j, k} \leq R 2^{-\alpha j}$ for every $j \in \mathbb{N}_{0}$. If $\alpha \leq 0$, it suffices to remark that $2^{0} d_{0,0}=\sup _{j \in \mathbb{N}_{0}, k \in\left\{0, \ldots, 2^{j}-1\right\}}\left|c_{j, k}\right|$.

2. If $\beta \geq 1$, then $\widetilde{A}(\alpha, \beta)=\Omega$.

Let us now define a distance on these auxiliary spaces.

Definition 3.9. Let $\alpha \in \mathbb{R}$ and $\beta \in\{-\infty\} \cup\left[0,+\infty\left[\right.\right.$. For $\vec{c}, \vec{c}^{\prime} \in \widetilde{A}(\alpha, \beta)$, we write

$$
\widetilde{\delta}_{\alpha, \beta}\left(\vec{c}, \vec{c}^{\prime}\right):=\inf \left\{C+C^{\prime}: C, C^{\prime} \geq 0 \text { and } \# \widetilde{E_{j}}(C, \alpha)\left(\vec{c}-\vec{c}^{\prime}\right) \leq C^{\prime} 2^{\beta j} \forall j \in \mathbb{N}_{0}\right\} .
$$

Lemma 3.10. Let $\alpha \in \mathbb{R}$ and $\beta \in\{-\infty\} \cup\left[0,+\infty\left[\right.\right.$. The space $\widetilde{A}(\alpha, \beta)$ is a vector space and $\widetilde{\delta}_{\alpha, \beta}$ is a distance on $\widetilde{A}(\alpha, \beta)$ which is invariant by translation and which satisfies

$$
\widetilde{\delta}_{\alpha, \beta}(\theta \vec{c}, \overrightarrow{0}) \leq \sup \{1,|\theta|\} \widetilde{\delta}_{\alpha, \beta}(\vec{c}, \overrightarrow{0})
$$

for all $\vec{c} \in \widetilde{A}(\alpha, \beta)$ and $\theta \in \mathbb{C}$.

Proof. By definition, it is clear that $\widetilde{\delta}_{\alpha, \beta}$ is translation invariant. For the other properties, it suffices to adapt the proof of Lemma 3.3 in [5] to the case of wavelet leaders.

Remark 3.11. It is direct to check that the distance defined by

$$
\widetilde{\delta}_{\alpha, \beta}^{*}\left(\vec{c}, \vec{c}^{\prime}\right):=\inf \left\{C \geq 0: \# \widetilde{E_{j}}(C, \alpha)\left(\vec{c}-\vec{c}^{\prime}\right) \leq C 2^{\beta j} \forall j \in \mathbb{N}_{0}\right\}
$$

leads to the same topology. 
If $\beta=-\infty$, then $\left(\widetilde{A}(\alpha, \beta), \widetilde{\delta}_{\alpha, \beta}\right)$ is the topological normed space $\left(C^{\alpha},\|\cdot\|_{C^{\alpha}}\right)$ if $\alpha>0$ and $\left(C^{0},\|\cdot\|_{C^{0}}\right)$ if $\alpha \leq 0$. Moreover, if $\beta \geq 1$, we have $\widetilde{\delta}_{\alpha, \beta} \leq 1$. In the following proposition, we also get more information about the topology in the case $\beta>1$.

For auxiliary spaces of $\mathcal{S}^{\nu}$, it is known that the topology defined by $\delta_{\alpha, \beta}$ is stronger than the pointwise topology; these topologies are equivalent when $\beta>1$. In the $\mathcal{L}^{\nu}$ case, the topology defined by $\widetilde{\delta}_{\alpha, \beta}$ is also stronger than the pointwise topology; moreover, it is stronger than the uniform topology, i.e. the topology defined by the norm of $C^{0}$. The equivalence with uniform topology happens when $\beta>1$.

Proposition 3.12. Let $\alpha \in \mathbb{R}$ and $\beta \in\{-\infty\} \cup[0,+\infty[$.

1. The addition is continuous on $\left(\widetilde{A}(\alpha, \beta), \widetilde{\delta}_{\alpha, \beta}\right)$.

2. The space $\left(\widetilde{A}(\alpha, \beta), \widetilde{\delta}_{\alpha, \beta}\right)$ has a stronger topology than the uniform topology. Moreover, every Cauchy sequence in $\left(\widetilde{A}(\alpha, \beta), \widetilde{\delta}_{\alpha, \beta}\right)$ is also a uniform Cauchy sequence.

3. If $\beta>1$, the topology defined by the distance $\widetilde{\delta}_{\alpha, \beta}$ is equivalent to uniform topology.

4. (a) If $B$ is a bounded set of $\left(\widetilde{A}(\alpha, \beta), \widetilde{\delta}_{\alpha, \beta}\right)$, then there exists $r>0$ such that

$$
\begin{array}{r}
B \subset\left\{\vec{c} \in \Omega: \#\left\{k \in\left\{0, \ldots, 2^{j}-1\right\}: d_{j, k} \geq r 2^{-\alpha j}\right\} \leq r 2^{\beta j} \forall j \in \mathbb{N}_{0}\right\} \\
\subset\left\{\vec{c} \in \Omega: \#\left\{k \in\left\{0, \ldots, 2^{j}-1\right\}: d_{j, k}>r 2^{-\alpha j}\right\} \leq r 2^{\beta j} \forall j \in \mathbb{N}_{0}\right\} .
\end{array}
$$

(b) Let $r, r^{\prime} \geq 0, \alpha^{\prime} \geq \alpha$ and $\beta^{\prime} \leq \beta$. The set

$$
B:=\left\{\vec{c} \in \Omega: \#\left\{k \in\left\{0, \ldots, 2^{j}-1\right\}: d_{j, k}>r 2^{-\alpha^{\prime} j}\right\} \leq r^{\prime} 2^{\beta^{\prime} j} \forall j \in \mathbb{N}_{0}\right\}
$$

is a bounded set of $\left(\widetilde{A}(\alpha, \beta), \widetilde{\delta}_{\alpha, \beta}\right)$. Moreover, $B$ is closed for the uniform convergence.

5. The space $\left(\widetilde{A}(\alpha, \beta), \widetilde{\delta}_{\alpha, \beta}\right)$ is a complete metric space.

Proof. 1. The first point is obvious using the triangular inequality with the distance $\widetilde{\delta}_{\alpha, \beta}$.

2. Let $\left(\vec{c}^{(m)}\right)_{m \in \mathbb{N}}$ be a sequence of elements of $\widetilde{A}(\alpha, \beta)$ which converges to $\vec{c}$ in $\left(\widetilde{A}(\alpha, \beta), \widetilde{\delta}_{\alpha, \beta}\right)$. If $\beta=-\infty$, it suffices to observe that we have

$$
\sup _{(j, k) \in \Lambda}\left|c_{j, k}^{(m)}-c_{j, k}\right|=\sup _{\lambda^{\prime} \subset \lambda(0,0)}\left|c_{\lambda^{\prime}}^{(m)}-c_{\lambda^{\prime}}\right| \leq \sup _{(j, k) \in \Lambda} 2^{\alpha j} \sup _{\lambda^{\prime} \subset \lambda(j, k)}\left|c_{\lambda^{\prime}}^{(m)}-c_{\lambda^{\prime}}\right|
$$

for every $m \in \mathbb{N}$. Let us consider now the case $\beta \geq 0$. Let $\varepsilon>0$ and $\eta:=\min \left\{\frac{1}{2}, \varepsilon\right\}$. There exists $M \in \mathbb{N}$ such that

$$
\#\left\{k \in\left\{0, \ldots, 2^{j}-1\right\}: \sup _{\lambda^{\prime} \subset \lambda(j, k)}\left|c_{\lambda^{\prime}}^{(m)}-c_{\lambda^{\prime}}\right| \geq \eta 2^{-\alpha j}\right\} \leq \eta 2^{\beta j}
$$

for all $j \in \mathbb{N}_{0}$ and $m \geq M$. Consequently, taking $j=0$, we obtain for all $m \geq M$,

$$
\sup _{\left(j_{0}, k_{0}\right) \in \Lambda}\left|c_{j_{0}, k_{0}}^{(m)}-c_{j_{0}, k_{0}}\right|=\sup _{\lambda^{\prime} \subset \lambda(0,0)}\left|c_{\lambda^{\prime}}^{(m)}-c_{\lambda^{\prime}}\right|<\eta \leq \varepsilon
$$

The proof is similar for Cauchy sequences.

3 . With the previous point, it only remains to show that the uniform topology is stronger than the topology defined by the distance $\widetilde{\delta}_{\alpha, \beta}$ (in the case $\beta>1$ ). Let $\left(\vec{c}^{(m)}\right)_{m \in \mathbb{N}}$ be a sequence of $\widetilde{A}(\alpha, \beta)=\Omega$ which converges uniformly to $\vec{c}$ and let $\varepsilon>0$. There exists $J \in \mathbb{N}_{0}$ such that $2^{j} \leq \varepsilon 2^{\beta j}$ for every $j \geq J$ because $\beta>1$ and then we have 


$$
\#\left\{k \in\left\{0, \ldots, 2^{j}-1\right\}: \sup _{\lambda^{\prime} \subset \lambda(j, k)}\left|c_{\lambda^{\prime}}^{(m)}-c_{\lambda^{\prime}}\right| \geq \varepsilon 2^{-\alpha j}\right\} \leq 2^{j} \leq \varepsilon 2^{\beta j}
$$

for every $j>J$ and $m \in \mathbb{N}$. Let us now fix $j \in\{0, \ldots, J\}$. Using the uniform convergence, there exists $M \in \mathbb{N}$ (which only depends on $\varepsilon$ ) such that

$$
\sup _{\lambda^{\prime} \subset \lambda(j, k)}\left|c_{\lambda^{\prime}}^{(m)}-c_{\lambda^{\prime}}\right|<\varepsilon 2^{-\alpha j}
$$

for every $k \in\left\{0, \ldots, 2^{j}-1\right\}$ and $m \geq M$. So, for every $m \geq M$, we have

$$
\#\left\{k \in\left\{0, \ldots, 2^{j}-1\right\}: \sup _{\lambda^{\prime} \subset \lambda(j, k)}\left|c_{\lambda^{\prime}}^{(m)}-c_{\lambda^{\prime}}\right| \geq \varepsilon 2^{-\alpha j}\right\}=0 \leq \varepsilon 2^{\beta j} .
$$

Thus $\left(\vec{c}^{(m)}\right)_{m \in \mathbb{N}}$ converges to $\vec{c}$ in $\left(\widetilde{A}(\alpha, \beta), \widetilde{\delta}_{\alpha, \beta}\right)$.

4. The proof is similar to the one in the $\mathcal{S}^{\nu}$ case (see Proposition 3.5 in [5]). Let us only show that $B$ is closed for the uniform convergence. Let $\left(\vec{c}^{(m)}\right)_{m \in \mathbb{N}}$ be a sequence of $B$ which converges uniformly to $\vec{c}$ and let $\varepsilon>0$. Then there exists $M \in \mathbb{N}$ such that

$$
\sup _{\lambda^{\prime} \subset \lambda(0,0)}\left|c_{\lambda^{\prime}}^{(m)}-c_{\lambda^{\prime}}\right|<\varepsilon
$$

for all $m \geq M$. Let us fix $j \in \mathbb{N}_{0}$ and $k \in\left\{0, \ldots, 2^{j}-1\right\}$. We have

$$
d_{j, k}>r 2^{-\alpha^{\prime} j} \Rightarrow d_{j, k}^{(M)}>r 2^{-\alpha^{\prime} j} .
$$

Otherwise, $d_{j, k}^{(M)} \leq r 2^{-\alpha^{\prime} j}$ and then, by taking $\varepsilon$ smaller if needed, we have

$$
r 2^{-\alpha^{\prime} j}<d_{j, k}-\varepsilon \leq \sup _{\lambda^{\prime} \subset \lambda(j, k)}\left|c_{\lambda^{\prime}}^{(M)}-c_{\lambda^{\prime}}\right|+d_{j, k}^{(M)}-\varepsilon \leq r 2^{-\alpha^{\prime} j}
$$

which is absurd. So $\vec{c} \in B$ because

$$
\#\left\{k \in\left\{0, \ldots, 2^{j}-1\right\}: d_{j, k}>r 2^{-\alpha^{\prime} j}\right\} \leq \#\left\{k \in\left\{0, \ldots, 2^{j}-1\right\}: d_{j, k}^{(M)}>r 2^{-\alpha^{\prime} j}\right\} \leq r^{\prime} 2^{\beta^{\prime} j} \quad \forall j \in \mathbb{N}_{0} .
$$

5. Since $\left(\widetilde{A}(\alpha, \beta), \widetilde{\delta}_{\alpha, \beta}\right)$ is a metric space, it only remains to show that if $\left(\vec{c}^{(m)}\right)_{m \in \mathbb{N}}$ is a Cauchy sequence in $\left(\widetilde{A}(\alpha, \beta), \widetilde{\delta}_{\alpha, \beta}\right)$, it converges in $\left(\widetilde{A}(\alpha, \beta), \widetilde{\delta}_{\alpha, \beta}\right)$. From the point 2 of this proposition, $\left(\vec{c}^{(m)}\right)_{m \in \mathbb{N}}$ is also a uniform Cauchy sequence and then it converges uniformly to $\vec{c}$. By hypothesis, if $\eta>0$, there exists $M \in \mathbb{N}$ such that

$$
\#\left\{k \in\left\{0, \ldots, 2^{j}-1\right\}: \sup _{\lambda^{\prime} \subset \lambda(j, k)}\left|c_{\lambda^{\prime}}^{(p)}-c_{\lambda^{\prime}}^{(q)}\right|>\eta 2^{-\alpha j}\right\} \leq \eta 2^{\beta j} \quad \forall j \in \mathbb{N}_{0}, \forall p, q \geq M
$$

It follows from the point 4 of this proposition that we have

$$
\#\left\{k \in\left\{0, \ldots, 2^{j}-1\right\}: \sup _{\lambda^{\prime} \subset \lambda(j, k)}\left|c_{\lambda^{\prime}}^{(p)}-c_{\lambda^{\prime}}\right|>\eta 2^{-\alpha j}\right\} \leq \eta 2^{\beta j} \quad \forall j \in \mathbb{N}_{0}, \forall p \geq M .
$$


Remark 3.13. If $\beta \in[0,1]$ and $\alpha>0$, the scalar multiplication $(\theta, \vec{c}) \in \mathbb{C} \times \widetilde{A}(\alpha, \beta) \mapsto \theta \vec{c} \in \widetilde{A}(\alpha, \beta)$ is not continuous and consequently, the space $\left(\widetilde{A}(\alpha, \beta), \widetilde{\delta}_{\alpha, \beta}\right)$ is not a topological vector space. Indeed, let $\vec{c}$ be the sequence of $\widetilde{A}(\alpha, \beta)$ defined by

$$
c_{j, k}:= \begin{cases}j 2^{-\alpha j} & \text { if } k \in\left\{0, \ldots,\left\lfloor 2^{\beta j}\right\rfloor-1\right\} \\ 0 & \text { if } k \in\left\{\left\lfloor 2^{\beta j}\right\rfloor, \ldots, 2^{j}-1\right\}\end{cases}
$$

for $j \in \mathbb{N}_{0}$. For large $j$, we have $\left\lfloor 2^{\beta(j+1)}\right\rfloor / 2 \leq\left\lfloor 2^{\beta j}\right\rfloor$ and then $d_{j, k}=c_{j, k}$ for such $j$ and $k \in\left\{0, \ldots, 2^{j}-1\right\}$. Following the proof of Proposition 3.5 in [5], the sequence $(\vec{c} / m)_{m \in \mathbb{N}}$ does not converge to $\overrightarrow{0}$ in $\left(\widetilde{A}(\alpha, \beta), \widetilde{\delta}_{\alpha, \beta}\right)$. This counterexample also shows that the topology defined by $\widetilde{\delta}_{\alpha, \beta}$ and the uniform topology are not equivalent for such $\beta$ and $\alpha$.

Let us end with some relations between auxiliary spaces. The second part is useful to obtain the continuity of the scalar multiplication in $\mathcal{L}^{\nu}$.

\section{Lemma 3.14.}

1. If $\alpha \geq \alpha^{\prime}$ and $\beta \leq \beta^{\prime}$, then

$$
\widetilde{A}(\alpha, \beta) \subset \widetilde{A}\left(\alpha^{\prime}, \beta^{\prime}\right) \quad \text { and } \quad \widetilde{\delta}_{\alpha^{\prime}, \beta^{\prime}} \leq \widetilde{\delta}_{\alpha, \beta}
$$

2. Let $\alpha^{\prime}>\alpha$ and $\beta^{\prime}<\beta$. If the sequence $\left(\theta_{m}\right)_{m \in \mathbb{N}}$ converges to $\theta$ in $\mathbb{C}$ and if the sequence $\left(\vec{c}^{(m)}\right)_{m \in \mathbb{N}}$ of $C^{0}$ converges to $\vec{c}$ in $\left(\widetilde{A}(\alpha, \beta), \widetilde{\delta}_{\alpha, \beta}\right)$ with $\vec{c} \in \widetilde{A}\left(\alpha^{\prime}, \beta^{\prime}\right)$, then the sequence $\left(\theta_{m} \vec{c}^{(m)}\right)_{m \in \mathbb{N}}$ converges to $\theta \vec{c}$ in $\left(\widetilde{A}(\alpha, \beta), \widetilde{\delta}_{\alpha, \beta}\right)$.

Proof. The first item is obvious. The second one is similar to the $\mathcal{S}^{\nu}$ case. Since the sequence $\left(\theta_{m}\right)_{m \in \mathbb{N}}$ converges to $\theta$ in $\mathbb{C}$, there exists $D>0$ such that $\left|\theta_{m}-\theta\right| \leq D$ for all $m \in \mathbb{N}$. We have

$$
\theta_{m} \vec{c}^{(m)}-\theta \vec{c}=\left(\theta_{m}-\theta\right)\left(\vec{c}^{(m)}-\vec{c}\right)-\theta\left(\vec{c}^{(m)}-\vec{c}\right)+\left(\theta_{m}-\theta\right) \vec{c}
$$

and then

$$
\widetilde{\delta}_{\alpha, \beta}\left(\theta_{m} \vec{c}^{(m)}, \theta \vec{c}\right) \leq \sup \{1, D\} \widetilde{\delta}_{\alpha, \beta}\left(\vec{c}^{(m)}, \vec{c}\right)+\sup \{1,|\theta|\} \widetilde{\delta}_{\alpha, \beta}\left(\vec{c}^{(m)}, \vec{c}\right)+\widetilde{\delta}_{\alpha, \beta}\left(\left(\theta_{m}-\theta\right) \vec{c}, \overrightarrow{0}\right)
$$

thanks to Lemma 3.10. The two first terms converge to 0 by using hypotheses and the first point of this lemma. Let us consider now the convergence of the third term. Since $\vec{c} \in \widetilde{A}\left(\alpha^{\prime}, \beta^{\prime}\right)$, there exist $C, C^{\prime} \geq 0$ such that

$$
\#\left\{k \in\left\{0, \ldots, 2^{j}-1\right\}: d_{j, k} \geq C 2^{-\alpha^{\prime} j}\right\} \leq C^{\prime} 2^{\beta^{\prime} j}
$$

for all $j \in \mathbb{N}_{0}$. Let $\eta>0$. Then there exists $J \in \mathbb{N}_{0}$ such that $D C 2^{-j\left(\alpha^{\prime}-\alpha\right)} \leq \eta$ and $C^{\prime} 2^{-j\left(\beta-\beta^{\prime}\right)} \leq \eta$ for all $j \geq J$. Consequently, we have, for all $j \geq J$ and $m \in \mathbb{N}$,

$$
\#\left\{k \in\left\{0, \ldots, 2^{j}-1\right\}:\left|\theta_{m}-\theta\right| d_{j, k} \geq \eta 2^{-\alpha j}\right\} \leq \eta 2^{\beta j}
$$

because $\left|\theta_{m}-\theta\right| \leq D$ for all $m \in \mathbb{N}$. Since the sequence $\left(\theta_{m}\right)_{m \in \mathbb{N}}$ converges to $\theta$ and $\vec{c} \in C^{0}$, there exists $M \in \mathbb{N}$ such that

$$
\left|\theta_{m}-\theta\right| d_{j, k}<\eta 2^{-\alpha j}
$$


for all $m \geq M, j \in\{0, \ldots, J-1\}$ and $k \in\left\{0, \ldots, 2^{j}-1\right\}$. Hence $\widetilde{\delta}_{\alpha, \beta}\left(\left(\theta_{m}-\theta\right) \vec{c}, \overrightarrow{0}\right) \leq 2 \eta$ for all $m \geq M$ and we get the conclusion.

\section{Remark 3.15.}

1. The assumption that the sequences belong to $C^{0}$ will not be restrictive because we know that $\mathcal{L}^{\nu} \subset C^{0}$.

2. If $\beta=\beta^{\prime}=-\infty$, this lemma remains true.

\subsection{Topology on $\mathcal{L}^{\nu}$ spaces}

By Proposition 3.7, we know that $\mathcal{L}^{\nu}$ is a countable intersection of auxiliary spaces. As in the case of $\mathcal{S}^{\nu}$ spaces, this description allows to obtain a structure of complete metric space on $\mathcal{L}^{\nu}$. Indeed, the idea is to use the following classical result of functional analysis (see for example [17]) to define a topology on $\mathcal{L}^{\nu}$.

Proposition 3.16. Let $E_{m}(m \in \mathbb{N})$ be spaces endowed with the topologies defined by the distances $d_{m}$ and set $E:=\bigcap_{m \in \mathbb{N}} E_{m}$. On $E$, let us consider the topology $\tau$ defined as follows: for every $e \in E$, a basis of neighbourhoods of $e$ is given by the family of sets

$$
\bigcap_{(m)}\left\{f \in E: d_{m}(e, f) \leq r_{m}\right\}
$$

where $r_{m}>0$ for every $m \in \mathbb{N}$ and $(m)$ means that it is an intersection on a finite number of values of $m$. Then, this topology satisfies the following properties.

1. For every $m \in \mathbb{N}$, the identity $i:(E, \tau) \rightarrow\left(E_{m}, d_{m}\right)$ is continuous and $\tau$ is the weakest topology on $E$ which verifies this property.

2. The topology $\tau$ is equivalent to the topology defined on $E$ by the distance $d$ given by

$$
d(e, f):=\sum_{m=1}^{+\infty} 2^{-m} \frac{d_{m}(e, f)}{1+d_{m}(e, f)}, \quad e, f \in E
$$

3. A sequence is a Cauchy sequence in $(E, \tau)$ if and only if it is a Cauchy sequence in $\left(E_{m}, d_{m}\right)$ for every $m \in \mathbb{N}$.

4. A sequence converges to $e$ in $(E, \tau)$ if and only if it converges to e in $\left(E_{m}, d_{m}\right)$ for every $m \in \mathbb{N}$.

Using some properties of the auxiliary spaces $\left(\widetilde{A}(\alpha, \beta), \widetilde{\delta}_{\alpha, \beta}\right)$ and Proposition 3.16, we can define a distance on the spaces $\mathcal{L}^{\nu}$ and obtain some additional information on these spaces.

Definition 3.17. Let $\boldsymbol{\alpha}:=\left(\alpha_{n}\right)_{n \in \mathbb{N}}$ be a dense sequence in $\mathbb{R}$ and $\varepsilon:=\left(\varepsilon_{m}\right)_{m \in \mathbb{N}}$ be a sequence of $] 0,+\infty[$ which converges to 0 . For $m, n \in \mathbb{N}$, we write

$$
\widetilde{\delta}_{m, n}:=\widetilde{\delta}_{\alpha_{n}, \nu\left(\alpha_{n}\right)+\varepsilon_{m}} \text { and } \quad \widetilde{A}(m, n):=\widetilde{A}\left(\alpha_{n}, \nu\left(\alpha_{n}\right)+\varepsilon_{m}\right) .
$$

Then, for $m \in \mathbb{N}$, we denote

$$
\widetilde{\delta}_{m}:=\sum_{n=1}^{+\infty} 2^{-n} \frac{\widetilde{\delta}_{m, n}}{1+\widetilde{\delta}_{m, n}}
$$


and

$$
\widetilde{\delta}_{\boldsymbol{\alpha}, \boldsymbol{\varepsilon}}:=\sum_{m=1}^{+\infty} 2^{-m} \widetilde{\delta}_{m} \quad\left(=\sum_{m=1}^{+\infty} \sum_{n=1}^{+\infty} 2^{-(m+n)} \frac{\widetilde{\delta}_{m, n}}{1+\widetilde{\delta}_{m, n}}\right) .
$$

A straightforward adaptation of the proof of Proposition 5.5 in [5] gives the following result.

Proposition 3.18. For every sequences $\boldsymbol{\alpha}$ and $\boldsymbol{\varepsilon}$ chosen as above, $\widetilde{\delta}_{\boldsymbol{\alpha}, \boldsymbol{\varepsilon}}$ is a distance on $\mathcal{L}^{\nu}$. All these distances define the same topology.

In view of this result, we write this distance $\widetilde{\delta}$ (on $\mathcal{L}^{\nu}$ ) independently of these sequences $\boldsymbol{\alpha}$ and $\varepsilon$. In fact, this result can be seen as a direct consequence of closed graph theorem since the metric defines a complete topological vector space, as we will see in Propositions 3.19 and 3.20.

Again, the next proposition is obtained by a direct modification of the proof of Theorem 5.7. in [5].

\section{Proposition 3.19.}

1. The topology defined by $\widetilde{\delta}$ on $\mathcal{L}^{\nu}$ is the weakest topology such that, for every $m, n \in \mathbb{N}$, the identity $i: \mathcal{L}^{\nu} \rightarrow \widetilde{A}(m, n)$ is continuous.

2. A sequence in $\mathcal{L}^{\nu}$ is a Cauchy sequence in $\left(\mathcal{L}^{\nu}, \widetilde{\delta}\right)$ if and only if, for every $m, n \in \mathbb{N}$, it is a Cauchy sequence in $\left(\widetilde{A}(m, n), \widetilde{\delta}_{m, n}\right)$.

3. A sequence in $\mathcal{L}^{\nu}$ converges in $\left(\mathcal{L}^{\nu}, \widetilde{\delta}\right)$ if and only if, for every $m, n \in \mathbb{N}$, it converges in $\left(\widetilde{A}(m, n), \widetilde{\delta}_{m, n}\right)$.

4. The space $\left(\mathcal{L}^{\nu}, \widetilde{\delta}\right)$ is a vector topological complete metric space and then a Baire space.

Proposition 3.20. If $\widetilde{\delta}_{1}$ and $\widetilde{\delta}_{2}$ define complete topologies on $\mathcal{L}^{\nu}$ which are stronger than the pointwise topology, then these topologies are equivalent.

Proof. It is a direct consequence of the closed graph theorem and the previous Proposition 3.19.

Remark 3.21. The inclusion $\mathcal{L}^{\nu} \subset C^{0}$ is continuous by combining Proposition 3.19 (item 3) and Proposition 3.12 (item 2).

In [8], it is proved that the definition of the $\mathcal{L}^{\nu}$ spaces is independent of the chosen basis wavelet basis. Therefore, these spaces can be seen independently as function spaces or as sequence spaces. Let us now show that the topology defined on $\mathcal{L}^{\nu}$ is a "good topology", in the sense that it is also independent of the chosen wavelet basis. This allows to consider the space $\left(\mathcal{L}^{\nu}, \widetilde{\delta}\right)$ as a topological function space.

Let us recall that operators which map an orthonormal wavelet basis in the Schwartz class into another orthonormal wavelet basis in the Schwartz class are quasidiagonal (see [22] for more details). Therefore, in order to check that a condition defined on wavelet coefficients is independent of the chosen wavelet basis (in the Schwartz class), one usually check the stronger property that it is invariant under the action of quasidiagonal operators.

Proposition 3.22. Let $A$ be a quasidiagonal operator. If $\alpha_{\min }>0$, the application

$$
A:\left(\mathcal{L}^{\nu}, \widetilde{\delta}\right) \rightarrow\left(\mathcal{L}^{\nu}, \widetilde{\delta}\right): \vec{c} \mapsto A \vec{c}
$$

is continuous. 
Proof. The result of [8] ensures that $A$ maps $\mathcal{L}^{\nu}$ into $\mathcal{L}^{\nu}$. As the operator $A$ is a linear operator between complete metrizable topological vector spaces whose topologies are stronger than the pointwise topology, the continuity is obtained using the closed graph theorem.

\subsection{Compact subsets of $\mathcal{L}^{\nu}$}

Let us continue with the characterization of compact subsets of $\left(\mathcal{L}^{\nu}, \widetilde{\delta}\right)$. This characterization will only hold if $\alpha_{\text {min }}>0$. It is in particular useful to prove the convergence of sequences in $\mathcal{L}^{\nu}$. For $m, n \in \mathbb{N}$, let $C(m, n)$ and $C^{\prime}(m, n)$ be positive constants and let us define

$$
\widetilde{K}_{m, n}:=\left\{\vec{c} \in \Omega: \#\left\{k \in\left\{0, \ldots, 2^{j}-1\right\}: d_{j, k}>C(m, n) 2^{-\alpha_{n} j}\right\} \leq C^{\prime}(m, n) 2^{\left(\nu\left(\alpha_{n}\right)+\varepsilon_{m}\right) j} \forall j \in \mathbb{N}_{0}\right\}
$$

(by taking the usual sequences of Proposition 3.7 and Definition 3.17). We write

$$
\widetilde{K}:=\bigcap_{m \in \mathbb{N}} \bigcap_{n \in \mathbb{N}} \widetilde{K}_{m, n}
$$

Here are some useful observations to obtain the characterization of compact subsets of $\left(\mathcal{L}^{\nu}, \widetilde{\delta}\right)$.

\section{Lemma 3.23.}

1. From all sequences of $\widetilde{K}$, we can extract a subsequence which converges pointwise.

2. Let $\alpha>0$ and $B$ be a bounded set of $\left(C^{\alpha},\|\cdot\|_{C^{\alpha}}\right)$. If $\left(\vec{c}^{(l)}\right)_{l \in \mathbb{N}}$ is a sequence of $B$ which converges pointwise to $\vec{c}$, then it converges uniformly to $\vec{c}$.

3. Let $\alpha_{0} \in \mathbb{R}, \beta_{0} \geq 0$ and $B$ be a bounded set of $\left(\widetilde{A}\left(\alpha_{0}, \beta_{0}\right), \widetilde{\delta}_{\alpha_{0}, \beta_{0}}\right)$. If $\left(\vec{c}^{(l)}\right)_{l \in \mathbb{N}}$ is a sequence of $B$ which converges uniformly to $\vec{c}$, then it converges to $\vec{c}$ in $\left(\widetilde{A}(\alpha, \beta), \widetilde{\delta}_{\alpha, \beta}\right)$ for all $\alpha$ and $\beta$ such that $\alpha<\alpha_{0}$ and $\beta>\beta_{0}$.

4. Let $\alpha_{0} \geq 0$ and $B$ a bounded set of $\left(C^{\alpha_{0}},\|\cdot\|_{C^{\alpha_{0}}}\right)$. If $\left(\vec{c}^{(l)}\right)_{l \in \mathbb{N}}$ is a sequence of $B$ which converges uniformly to $\vec{c}$, then it converges to $\vec{c}$ in $\left(C^{\alpha},\|\cdot\|_{C^{\alpha}}\right)$ for all $\alpha<\alpha_{0}$.

Proof. 1. Let $\left(\vec{c}^{(l)}\right)_{l \in \mathbb{N}}$ be a sequence of $\widetilde{K}$. There exists $n \in \mathbb{N}_{0}$ such that $\alpha_{n}<\alpha_{\text {min }}$ and then we have

$$
\left|c_{j, k}^{(l)}\right| \leq 2^{-\alpha_{n}} C(m, n) \quad \forall l \in \mathbb{N}, j \in \mathbb{N}_{0}, k \in\left\{0, \ldots, 2^{j}-1\right\}
$$

This means that the sequence $\left(\vec{c}^{(l)}\right)_{l \in \mathbb{N}}$ is pointwise bounded in $\mathbb{C}$ and we can thus extract a pointwise convergent subsequence.

2. By hypothesis, there exists $R>0$ such that $\left|c_{j, k}^{(l)}-c_{j, k}\right| \leq R 2^{-\alpha j}$ for every $j \in \mathbb{N}_{0}, k \in\left\{0, \ldots, 2^{j}-1\right\}$ and every $l \in \mathbb{N}$. Let $\eta>0$. On one hand, since $\alpha>0$, there exists $J \in \mathbb{N}_{0}$ such that $R 2^{-\alpha j}<\eta$ for every $j>J$ and then

$$
\left|c_{j, k}^{(l)}-c_{j, k}\right|<\eta \quad \forall l \in \mathbb{N}, j>J, k \in\left\{0, \ldots, 2^{j}-1\right\} .
$$

On the other hand, thanks to the pointwise convergence, there exists $L \in \mathbb{N}$ (which only depends on $\eta$ ) such that

$$
\sup _{j \in\{0, \ldots, J\}} \sup _{k \in\left\{0, \ldots, 2^{j}-1\right\}}\left|c_{j, k}^{(l)}-c_{j, k}\right|<\eta \quad \forall l \geq L .
$$


Thus

$$
\sup _{j \in \mathbb{N}_{0}} \sup _{k \in\left\{0, \ldots, 2^{j}-1\right\}}\left|c_{j, k}^{(l)}-c_{j, k}\right|<\eta \quad \forall l \geq L
$$

3. Since the sequence $\left(\vec{c}^{(l)}-\vec{c}\right)_{l \in \mathbb{N}}$ is bounded in $\left(\widetilde{A}\left(\alpha_{0}, \beta_{0}\right), \widetilde{\delta}_{\alpha_{0}, \beta_{0}}\right)$, there exist $R, R^{\prime} \geq 0$ such that

$$
\#\left\{k \in\left\{0, \ldots, 2^{j}-1\right\}: \sup _{\lambda^{\prime} \subset \lambda(j, k)}\left|c_{\lambda^{\prime}}^{(l)}-c_{\lambda^{\prime}}\right|>R 2^{-\alpha_{0} j}\right\} \leq R^{\prime} 2^{\beta_{0} j} \quad \forall j \in \mathbb{N}_{0}, l \in \mathbb{N},
$$

using Lemma 3.12 (item 4). Let $\eta>0$. Since $\alpha<\alpha_{0}$ and $\beta>\beta_{0}$, there exists $J \in \mathbb{N}_{0}$ such that $R 2^{-\alpha_{0} j}<$ $\eta 2^{-\alpha j}$ and $R^{\prime} 2^{\beta_{0} j}<\eta 2^{\beta j}$ for every $j>J$ and then

$$
\#\left\{k \in\left\{0, \ldots, 2^{j}-1\right\}: \sup _{\lambda^{\prime} \subset \lambda(j, k)}\left|c_{\lambda^{\prime}}^{(l)}-c_{\lambda^{\prime}}\right| \geq \eta 2^{-\alpha j}\right\} \leq \eta 2^{\beta j} \quad \forall l \in \mathbb{N}, j>J .
$$

Moreover, thanks to the uniform convergence, there exists $L \in \mathbb{N}$ (which only depends on $\eta$ ) such that

$$
\sup _{\lambda^{\prime} \subset \lambda(j, k)}\left|c_{\lambda^{\prime}}^{(l)}-c_{\lambda^{\prime}}\right|<\eta 2^{-\alpha j} \quad \forall j \in\{0, \ldots, J\}, k \in\left\{0, \ldots, 2^{j}-1\right\}, l \geq L
$$

and then

$$
\#\left\{k \in\left\{0, \ldots, 2^{j}-1\right\}: \sup _{\lambda^{\prime} \subset \lambda(j, k)}\left|c_{\lambda^{\prime}}^{(l)}-c_{\lambda^{\prime}}\right| \geq \eta 2^{-\alpha j}\right\}=0 \leq \eta 2^{\beta j} \quad \forall j \in\{0, \ldots, J\}, l \geq L .
$$

Thus, we have $\widetilde{\delta}_{\alpha, \beta}\left(\vec{c}^{(l)}, \vec{c}\right) \leq 2 \eta$ for every $l \geq L$.

4. The proof of this item is similar to the two previous ones.

Proposition 3.24. Let us assume that $\alpha_{\min }>0$. A set is a compact subset of $\left(\mathcal{L}^{\nu}, \widetilde{\delta}\right)$ if and only if it is closed in $\left(\mathcal{L}^{\nu}, \widetilde{\delta}\right)$ and included in some $\widetilde{K}$.

Proof. Since any compact set of a metric space is closed and bounded, the condition is obviously necessary.

Let us show that $\widetilde{K}$ is compact. Let $\left(\vec{c}^{(l)}\right)_{l \in \mathbb{N}}$ be a sequence of $\widetilde{K}$. By Lemma 3.23 (item 1), we can extract a subsequence which converges pointwise. Let us note again $\left(\vec{c}^{(l)}\right)_{l \in \mathbb{N}}$ the subsequence and $\vec{c}$ its pointwise limit. Let us show that $\left(\vec{c}^{(l)}\right)_{l \in \mathbb{N}}$ converges to $\vec{c}$ in $\left(\mathcal{L}^{\nu}, \widetilde{\delta}\right)$.

As $\alpha_{\min }>0$, there exists $n_{0} \in \mathbb{N}$ such that $0<\alpha_{n_{0}}<\alpha_{\min }$. By construction, $\vec{c}^{(l)} \in \widetilde{K}_{m, n_{0}}$ for all $l \in \mathbb{N}$ and $m \in \mathbb{N}$ and we know that $\widetilde{K}_{m, n_{0}}$ is bounded in $\left(C^{\alpha_{n_{0}}},\|\cdot\|_{C^{\alpha_{n_{0}}}}\right)$. Using Lemma 3.23 (item 2), we get that $\left(\vec{c}^{(l)}\right)_{l \in \mathbb{N}}$ converges uniformly to $\vec{c}$.

Let $\alpha \in \mathbb{R}$ and $\varepsilon>0$. If $\nu(\alpha) \in \mathbb{R}$, the right-continuity of $\nu$ gives $n, m \in \mathbb{N}$ such that

$$
\varepsilon_{m} \leq \varepsilon, \quad \alpha_{n}>\alpha \text { and } \quad \nu\left(\alpha_{n}\right)+\varepsilon_{m}<\nu(\alpha)+\varepsilon .
$$

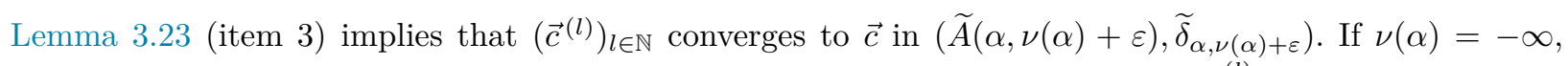
there exists $n \in \mathbb{N}$ such that $\alpha_{n}>\alpha$ and $\nu\left(\alpha_{n}\right)=-\infty$. By Lemma 3.23 (item 4$),\left(\vec{c}^{(l)}\right)_{l \in \mathbb{N}}$ converges to $\vec{c}$ in

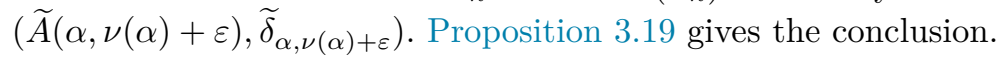

In fact, we also have obtained within this last proof the following result.

Corollary 3.25. Every sequence of $\widetilde{K}$ which converges pointwise converges also in $\left(\mathcal{L}^{\nu}, \widetilde{\delta}\right)$ to an element of $\widetilde{K}$. 
Remark 3.26. The characterization is not longer valid in the case $\alpha_{\min }=0$. Indeed, let $\nu$ be the admissible profile defined by

$$
\nu(\alpha):= \begin{cases}-\infty & \text { if } \alpha<0 \\ 1 & \text { if } \alpha \geq 0 .\end{cases}
$$

It is direct to see that $\mathcal{L}^{\nu}=C^{0}$. If we assume that we have this characterization, then the unit ball of $C^{0}$ would be compact and therefore the space would be finite dimensional. This leads to a contradiction.

\subsection{Separability}

As for the characterization of the compact subsets of $\mathcal{L}^{\nu}$, we have to consider separately two cases: $\alpha_{\min }>0$ and $\alpha_{\min }=0$. Let us start with a first difference described in the following lemma.

Lemma 3.27. If $\vec{c} \in \Omega$, let $\left(\vec{c}^{N}\right)_{N \in \mathbb{N}_{0}}$ be the sequence of $\Omega$ defined by

$$
c_{j, k}^{N}:= \begin{cases}c_{j, k} & \text { if } j \leq N \text { and } k \in\left\{0, \ldots, 2^{j}-1\right\} \\ 0 & \text { if } j>N \text { and } k \in\left\{0, \ldots, 2^{j}-1\right\}\end{cases}
$$

for every $N \in \mathbb{N}_{0}$.

1. If $\alpha_{\min }>0,\left(\vec{c}^{N}\right)_{N \in \mathbb{N}_{0}}$ converges to $\vec{c}$ in $\left(\mathcal{L}^{\nu}, \widetilde{\delta}\right)$ for all $\vec{c} \in \mathcal{L}^{\nu}$.

2. If $\alpha_{\min }=0$, there exists $\vec{c} \in \mathcal{L}^{\nu}$ such that $\left(\vec{c}^{N}\right)_{N \in \mathbb{N}_{0}}$ does not converge to $\vec{c}$ in $\left(\mathcal{L}^{\nu}, \widetilde{\delta}\right)$.

Proof. 1. Since the characterization of compacts of $\mathcal{L}^{\nu}$ (when $\alpha_{\min }>0$ ) is similar to the one in the $\mathcal{S}^{\nu}$ case, the proof of this first point only needs clear simple adaptations of Lemma 6.3 in [5] with wavelet leaders.

2. We suppose now that $\alpha_{\text {min }}=0$ and we consider the sequence $\vec{c}$ where at each scale $j \in \mathbb{N}_{0}$, the element $c_{j, 0}$ is set equal to 1 and the others to 0 . For every $j \in \mathbb{N}_{0}$ and $k \in\left\{0, \ldots, 2^{j}-1\right\}$, we have $d_{j, k}=c_{j, k}$. Using the assumption $\alpha_{\min }=0$, it is trivial to check that $\vec{c}$ belongs to $\mathcal{L}^{\nu}$. Moreover, we know that the space $\left(\mathcal{L}^{\nu}, \widetilde{\delta}\right)$ has a stronger topology than the topology of the uniform convergence. We get then the conclusion because

$$
\sup _{j \in \mathbb{N}_{0}} \sup _{k \in\left\{0, \ldots, 2^{j}-1\right\}}\left|c_{j, k}-c_{j, k}^{N}\right|=1
$$

for every $N \in \mathbb{N}_{0}$.

Let us begin by studying the separability of $\mathcal{L}^{\nu}$ when $\alpha_{\min }>0$.

Lemma 3.28. Let $B$ be a pointwise bounded set of sequences and let us assume that there exists $N \in \mathbb{N}_{0}$ such that

$$
\forall \vec{c} \in B, \forall j>N, \forall k \in\left\{0, \ldots, 2^{j}-1\right\}, \quad c_{j, k}=0 .
$$

If $\alpha_{\min }>0$, then $B$ is included in a compact subset of $\mathcal{L}^{\nu}$.

Proof. Since $B$ is a pointwise bounded set, there exists a constant $C>0$ such that

$$
\sup _{j \in\{0, \ldots, N\}} \sup _{k \in\left\{0, \ldots, 2^{j}-1\right\}}\left|c_{j, k}\right| \leq C \quad \forall \vec{c} \in B .
$$


If $\vec{c} \in B$, then $c_{j, k}=0$ and therefore $d_{j, k}=0$ for every $j>N, k \in\left\{0, \ldots, 2^{j}-1\right\}$. So, for every $j \in\{0, \ldots, N\}, k \in\left\{0, \ldots, 2^{j}-1\right\}$ and $n \in \mathbb{N}$, we have

$$
2^{\alpha_{n} j} d_{j, k} \leq 2^{\alpha_{n} j} \sup _{j^{\prime} \in\{0, \ldots, N\}} \sup _{k^{\prime} \in\left\{0, \ldots, 2^{j^{\prime}}-1\right\}}\left|c_{j^{\prime}, k^{\prime}}\right| \leq C 2^{\alpha_{n} j}
$$

and we get that for any constant $C^{\prime}(m, n) \geq 0$,

$$
\begin{aligned}
& B \subset \bigcap_{m \in \mathbb{N}} \bigcap_{n \in \mathbb{N}}\{\vec{a} \in \Omega: \#\left\{k \in\left\{0, \ldots, 2^{j}-1\right\}: \sup _{\lambda^{\prime} \subset \lambda(j, k)}\left|a_{\lambda^{\prime}}\right|>C(m, n) 2^{-\alpha_{n} j}\right\} \\
&\left.\leq C^{\prime}(m, n) 2^{\left(\nu\left(\alpha_{n}\right)+\varepsilon_{m}\right) j} \forall j \in \mathbb{N}_{0}\right\}
\end{aligned}
$$

with $C(m, n)=C \sup _{j<N} \sup _{k \in\left\{0, \ldots, 2^{j}-1\right\}} 2^{\alpha_{n} j}$.

Proposition 3.29. If $\alpha_{\min }>0$, the metric space $\left(\mathcal{L}^{\nu}, \widetilde{\delta}\right)$ is separable.

Proof. If $\mathbb{Q}$ denotes the set of the complex numbers with rational real and imaginary parts, the set

$$
U:=\left\{\vec{c} \in \Omega: c_{j, k} \in \mathbb{Q} \text { and } \exists N \in \mathbb{N}_{0} \text { such that } c_{j, k}=0 \forall j>N, k \in\left\{0, \ldots, 2^{j}-1\right\}\right\}
$$

is dense in $\left(\mathcal{L}^{\nu}, \widetilde{\delta}\right)$, using the density of $\mathbb{Q}$ in $\mathbb{C}$, Lemma 3.27, Lemma 3.28 and Corollary 3.25.

Let us consider now the case where the admissible profile $\nu$ is such that $\alpha_{\min }=0$. The previous result is no longer valid. Indeed, with the admissible profile considered in Remark 3.26, the space $\mathcal{L}^{\nu}$ is $C^{0}$ which is not separable. More generally, we have the following property.

Proposition 3.30. If $\alpha_{\min }=0$, the metric space $\left(\mathcal{L}^{\nu}, \widetilde{\delta}\right)$ is not separable.

Proof. This result uses classical consideration concerning sup-norms. Indeed, let us consider the uncountable set $A$ of sequences $\vec{c}$ of $\Omega$ such that for each scale $j \in \mathbb{N}_{0}, c_{j, 0} \in\{0,1\}$ and the other coefficients are equal to 0 . Using the assumption on $\alpha_{\text {min }}$, we easily prove that $A$ is a subset of $\mathcal{L}^{\nu}$. Moreover, $\left\|\vec{c}-\vec{c}^{\prime}\right\|_{C^{0}}=1$ for all distinct elements $\vec{c}$ and $\vec{c}^{\prime}$ of $A$.

Let $D$ be a dense subset of $\left(\mathcal{L}^{\nu}, \widetilde{\delta}\right)$. For every $\vec{c} \in A$, there exists a sequence $\left(\vec{c}^{(m)}\right)_{m \in \mathbb{N}}$ of elements of $D$ which converges in $\left(\mathcal{L}^{\nu}, \widetilde{\delta}\right)$ to $\vec{c} \in \mathcal{L}^{\nu}$. From Proposition 3.19, the convergence also holds in $C^{0}$. Consequently, there exists $M \in \mathbb{N}$ such that

$$
\left\|\vec{c}-\vec{c}^{(m)}\right\|_{C^{0}}<\frac{1}{2} \quad \forall m \geq M
$$

In particular, there exists $\vec{a} \in D$ such that

$$
\|\vec{c}-\vec{a}\|_{C^{0}}<\frac{1}{2}
$$

Since the $C^{0}$ norm between two distinct elements of $A$ is equal to $1, D$ must contain at least as many elements as $A$ and cannot be countable. 


\section{Generic property in $\mathcal{L}^{\nu}$ spaces}

In this section, we study the form of the wavelet leaders profile of most of the sequences in $\mathcal{L}^{\nu}$. Let us recall that any wavelet leaders profile $\widetilde{\nu}_{\vec{c}}$ of $\vec{c}$ takes values in $\{-\infty\} \cup[0,1]$ and that there exists $\alpha_{\text {min }} \geq 0$ such that $\widetilde{\nu}_{\vec{c}}(\alpha)=-\infty$ for every $\alpha<\alpha_{\min }$. Moreover, $\widetilde{\nu}_{\vec{c}}$ is an increasing and right-continuous function. An admissible profile is then a function $\nu$ which satisfies these properties. In the next result, we show that these properties characterize entirely the wavelet leaders profile.

Proposition 4.1. Any admissible profile is the wavelet leaders profile of a sequence of $C^{0}$.

Proof. Let $\nu$ be an admissible profile and let us consider a sequence $\left(\alpha_{n}\right)_{n \in \mathbb{N}}$ whose elements form a dense subset of $\left[\alpha_{\min },+\infty\right.$ [. Using the right-continuity of $\nu$ and of the wavelet leaders profile, it suffices to construct a sequence $\vec{c} \in C^{0}$ such that $\widetilde{\nu}_{\vec{c}}\left(\alpha_{n}\right)=\nu\left(\alpha_{n}\right)$ for every $n \in \mathbb{N}$ and such that $\widetilde{\nu}_{\vec{c}}(\alpha)=-\infty$ if $\alpha<\alpha_{\min }$. For every $n \in \mathbb{N}$, let us first construct a sequence $\vec{c}^{(n)}$ such that $\widetilde{\nu}_{\vec{c}(n)}\left(\alpha_{n}\right)=\nu\left(\alpha_{n}\right)$.

If $n \in \mathbb{N}$ is such that $\nu\left(\alpha_{n}\right)=1$, we consider the sequence $\vec{c}^{(n)}$ defined by

$$
c_{j, k}^{(n)}:=2^{-\alpha_{n}(j+n)}, \quad \forall j \in \mathbb{N}_{0}, k \in\left\{0, \ldots, 2^{j}-1\right\} .
$$

Then, $\widetilde{\nu}_{\vec{c}(n)}\left(\alpha_{n}\right)=1$. Assume now that $n \in \mathbb{N}$ is such that $\nu\left(\alpha_{n}\right)<1$. If $\nu\left(\alpha_{n}\right)=0$, we set $J_{n}=0$. If $\left.\nu\left(\alpha_{n}\right) \in\right] 0,1\left[\right.$, let $J_{n}$ be the smallest integer such that

$$
2^{\nu\left(\alpha_{n}\right) J_{n}} \geq \frac{2}{2^{1-\nu\left(\alpha_{n}\right)}-1} .
$$

Then, for every $j \geq J_{n}$, we have $2^{\nu\left(\alpha_{n}\right) j} \leq 2\left(2^{\nu\left(\alpha_{n}\right)(j-1)}-1\right)$, hence

$$
\left\lfloor 2^{\nu\left(\alpha_{n}\right) j}\right\rfloor \leq 2\left\lfloor 2^{\nu\left(\alpha_{n}\right)(j-1)}\right\rfloor .
$$

Let us define $\vec{c}^{(n)}$ as follows: if $j<J_{n}$, we set $c_{j, k}^{(n)}:=0$ and if $j \geq J_{n}$, we set

$$
c_{j, k}^{(n)}:= \begin{cases}2^{-\alpha_{n}(j+n)} & \text { for }\left\lfloor 2^{\nu\left(\alpha_{n}\right) j}\right\rfloor \text { values of } k, \\ 0 & \text { otherwise, }\end{cases}
$$

where the positions of the $k \in\left\{0, \ldots, 2^{j}-1\right\}$ such that $c_{j, k}^{(n)}=2^{-\alpha_{n}(j+n)}$ are chosen first to fill entirely dyadic cubes of scale $j-1$ whose coefficients equal $2^{-\alpha_{n}(j-1+n)}$ (this is ensured by (2)). It follows that if $j \geq J_{n}$, the wavelet leaders are the wavelet coefficients. In particular, one has $\widetilde{\nu}_{\vec{c}(n)}\left(\alpha_{n}\right)=\nu\left(\alpha_{n}\right)$.

Now, we consider the sequence $\vec{c}$ defined using the sequences $\vec{c}^{(n)}, n \in \mathbb{N}$, as follows: we set $c_{0,0}:=0$ and for every $j \in \mathbb{N}, k \in\left\{0, \ldots, 2^{j}-1\right\}$, we set

$$
c_{j, k}:= \begin{cases}c_{j-1, k-2^{j-1}}^{(1)} & \text { if } k \in\left\{2^{j-1}, \ldots, 2^{j}-1\right\}, \\ c_{j-2, k-2^{j-2}}^{(2)} & \text { if } k \in\left\{2^{j-2}, \ldots, 2^{j-1}-1\right\}, \\ \vdots & \text { if } k \in\{2,3\}, \\ c_{1, k-2}^{(j-1)} & \text { if } k=1, \\ c_{0,0}^{(j)} & \text { if } k=0 . \\ 0 & \text {. }\end{cases}
$$

Clearly, we have $\left|c_{j, k}\right| \leq 2^{-\alpha_{\min } j}$ for every $j \in \mathbb{N}_{0}, k \in\left\{0, \ldots, 2^{j}-1\right\}$, and therefore $\vec{c} \in C^{\alpha_{\min }} \subset C^{0}$. In particular, if $\alpha<\alpha_{\min }$, then $\widetilde{\nu}_{\vec{c}}(\alpha)=-\infty$. 
Let us now show that for every $n \in \mathbb{N}$, we have $\widetilde{\nu}_{\vec{c}}\left(\alpha_{n}\right)=\nu\left(\alpha_{n}\right)$. By construction, it is clear that $\widetilde{\nu}_{\vec{c}}\left(\alpha_{n}\right) \geq \widetilde{\nu}_{\vec{c}(n)}\left(\alpha_{n}\right)=\nu\left(\alpha_{n}\right)$. In particular, if $\nu\left(\alpha_{n}\right)=1$, then $\widetilde{\nu}_{\vec{c}}\left(\alpha_{n}\right)=1$. Let us assume that $\nu\left(\alpha_{n}\right)<1$ and let us prove that $\widetilde{\nu}_{\vec{c}}\left(\alpha_{n}\right) \leq \nu\left(\alpha_{n}\right)$. Using the right-continuity of $\nu$, we fix $\varepsilon>0$ such that $\nu\left(\alpha_{n}+\varepsilon\right)<1$. At a given scale $j \geq n$, we have to take into consideration the sequences $\vec{c}^{(1)}, \ldots, \vec{c}^{(j)}$ and the restricted wavelet leaders corresponding to $k=0$. More precisely,

$$
\#\left\{\lambda \in \Lambda_{j}: d_{\lambda} \geq 2^{-\left(\alpha_{n}+\varepsilon\right) j}\right\} \leq \sum_{m=1}^{j} \#\left\{\lambda \in \Lambda_{j-m}: d_{\lambda}^{(m)} \geq 2^{-\left(\alpha_{n}+\varepsilon\right) j}\right\}+1,
$$

where we have added the case $k=0$. By construction, we know that if $\alpha_{m}>\alpha_{n}+\varepsilon$, we have

$$
\#\left\{\lambda \in \Lambda_{j-m}: d_{\lambda}^{(m)} \geq 2^{-\left(\alpha_{n}+\varepsilon\right) j}\right\}=0 .
$$

Assume then that $\alpha_{m} \leq \alpha_{n}+\varepsilon$. If $j-m \geq J_{m}$, we have $d_{j-m, k}^{(m)}=c_{j-m, k}^{(m)}$ and it follows that

$$
\#\left\{\lambda \in \Lambda_{j-m}: d_{\lambda}^{(m)} \geq 2^{-\left(\alpha_{n}+\varepsilon\right) j}\right\} \leq 2^{\nu\left(\alpha_{m}\right)(j-m)} \leq 2^{\nu\left(\alpha_{n}+\varepsilon\right) j} .
$$

If $j-m \leq J_{m}$, we have

$$
\begin{aligned}
\#\left\{\lambda \in \Lambda_{j-m}: d_{\lambda}^{(m)} \geq 2^{-\left(\alpha_{n}+\varepsilon\right) j}\right\} & \leq\left\lfloor 2^{\nu\left(\alpha_{m}\right) J_{m}}\right\rfloor \\
& \leq 2\left\lfloor 2^{\nu\left(\alpha_{m}\right)\left(J_{m}-1\right)}\right\rfloor \\
& \leq \frac{4}{2^{1-\nu\left(\alpha_{m}\right)}-1} \\
& \leq \frac{4}{2^{1-\nu\left(\alpha_{n}+\varepsilon\right)}-1}
\end{aligned}
$$

using (1) and (2). If $j$ is large enough, we have

$$
\frac{4}{2^{1-\nu\left(\alpha_{n}+\varepsilon\right)}-1} \leq 2^{\nu\left(\alpha_{n}+\varepsilon\right) j}
$$

and it follows that

$$
\#\left\{\lambda \in \Lambda_{j}: d_{\lambda} \geq 2^{-\left(\alpha_{n}+\varepsilon\right) j}\right\} \leq j 2^{\nu\left(\alpha_{n}+\varepsilon\right) j}+1
$$

Therefore,

$$
\widetilde{\nu}_{\vec{c}}\left(\alpha_{n}\right) \leq \lim _{\varepsilon \rightarrow 0^{+}} \nu\left(\alpha_{n}+\varepsilon\right)=\nu\left(\alpha_{n}\right)
$$

using the right-continuity of $\nu$. The conclusion follows.

The next result shows that for most of the sequences in $\mathcal{L}^{\nu}$, the wavelet leaders profile is equal to the profile $\nu$. Let us recall that a subset $A$ of a Baire space $X$ is called residual (or comeager) if $A$ contains a countable intersection of dense open sets of $X$, or equivalently if $X \backslash A$ is of first category (see [17] for example).

Theorem 4.2. Let $\nu$ be an admissible profile such that $\alpha_{\min }>0$. The set of sequences $\vec{c} \in \mathcal{L}^{\nu}$ such that $\widetilde{\nu}_{\vec{c}}=\nu$ is residual in $\left(\mathcal{L}^{\nu}, \widetilde{\delta}\right)$. 
Proof. Using Proposition 4.1, we can consider $\vec{z} \in \mathcal{L}^{\nu}$ such that $\widetilde{\nu}_{\vec{z}}=\nu$. Then, using the definition of $\widetilde{\nu}_{\vec{z}}$, for every $m, n \in \mathbb{N}$, there exists an infinite set $J_{m, n}$ such that

$$
\#\left\{\lambda \in \Lambda_{j}: \sup _{\lambda^{\prime} \subset \lambda}\left|z_{\lambda^{\prime}}\right| \geq 2^{-\left(\alpha_{n}+2 \varepsilon_{m}\right) j}\right\} \geq 2^{\left(\nu\left(\alpha_{n}\right)-\varepsilon_{m}\right) j}, \quad \forall j \in J_{m, n} .
$$

From Proposition 3.29, we know that $\mathcal{L}^{\nu}$ is separable, and more precisely, that the set $U=\left\{\vec{y}^{(l)}: l \in \mathbb{N}\right\}$ formed by the rational finite sequences is dense in $\mathcal{L}^{\nu}$. Moreover, by construction, for every $l \in \mathbb{N}$, there exists $j_{l} \in \mathbb{N}_{0}$ such that $y_{\lambda}^{(l)}=0$ for every $\lambda \in \Lambda_{j}$ with $j \geq j_{l}$. For every $m, n, l \in \mathbb{N}$, we fix $j_{m, n, l} \in J_{m, n}$ such that

$$
j_{m, n, l} \geq j_{l} \quad \text { and } \quad \varepsilon_{m} j_{m, n, l}>1
$$

For every $m, n, L \in \mathbb{N}$, let us consider the set $U_{m, n, L}$ defined by

$$
U_{m, n, L}:=\bigcup_{l \geq L} \widetilde{B}_{m, n, l}
$$

where $\widetilde{B}_{m, n, l}$ is the open ball in the auxiliary space $\widetilde{A}\left(\alpha_{n}, \nu\left(\alpha_{n}\right)+\varepsilon_{m}\right)$ formed by the sequences $\vec{c} \in \mathcal{L}^{\nu}$ such that

$$
\inf \left\{C>0: \#\left\{\lambda \in \Lambda_{j}: \sup _{\lambda^{\prime} \subset \lambda}\left|c_{\lambda^{\prime}}-y_{\lambda^{\prime}}^{(l)}\right| \geq C 2^{-\alpha_{n} j}\right\} \leq C 2^{\left(\nu\left(\alpha_{n}\right)+\varepsilon_{m}\right) j}, \forall j \in \mathbb{N}_{0}\right\}<2^{-3 \varepsilon_{m} j_{m, n, l}} .
$$

Remark that, for every $m, n, L \in \mathbb{N}$, the set $U_{m, n, L}$ is dense in $\mathcal{L}^{\nu}$ since it contains the sequences $\vec{y}^{(l)}, l \geq L$. Finally, the set

$$
W:=\bigcap_{m, n, L \in \mathbb{N}} U_{m, n, L}
$$

is a countable intersection of dense open sets of $\mathcal{L}^{\nu}$.

Let us show that if $\vec{c} \in W$, then $\widetilde{\nu}_{\vec{c}}=\nu$. Since $W \subset \mathcal{L}^{\nu}$, we already know that $\widetilde{\nu}_{\vec{c}} \leq \nu$. For every $m, n, L \in \mathbb{N}$, there is $l \geq L$ such that $\vec{c} \in \widetilde{B}_{m, n, l}$, so

$$
\#\left\{\lambda \in \Lambda_{j}: \sup _{\lambda^{\prime} \subset \lambda}\left|c_{\lambda^{\prime}}-y_{\lambda^{\prime}}^{(l)}\right| \geq 2^{-3 \varepsilon_{m} j_{m, n, l}} 2^{-\alpha_{n} j}\right\} \leq 2^{-3 \varepsilon_{m} j_{m, n, l}} 2^{\left(\nu\left(\alpha_{n}\right)+\varepsilon_{m}\right) j}
$$

for every $j \in \mathbb{N}_{0}$. Then, for $j=j_{m, n, l}$, we obtain

$$
\begin{aligned}
& \#\left\{\lambda \in \Lambda_{j}: \sup _{\lambda^{\prime} \subset \lambda}\left|c_{\lambda^{\prime}}\right| \geq 2^{-\left(\alpha_{n}+3 \varepsilon_{m}\right) j}\right\} \\
& \geq \#\left\{\lambda \in \Lambda_{j}: \sup _{\lambda^{\prime} \subset \lambda}\left|y_{\lambda^{\prime}}^{l}\right|-\sup _{\lambda^{\prime} \subset \lambda}\left|c_{\lambda^{\prime}}-y_{\lambda^{\prime}}^{(l)}\right| \geq 2^{-\left(\alpha_{n}+3 \varepsilon_{m}\right) j}\right\} \\
& \geq \#\left\{\lambda \in \Lambda_{j}: \sup _{\lambda^{\prime} \subset \lambda}\left|y_{\lambda^{\prime}}^{(l)}\right| \geq 2 \cdot 2^{-\left(\alpha_{n}+3 \varepsilon_{m}\right) j} \text { and } \sup _{\lambda^{\prime} \subset \lambda}\left|c_{\lambda^{\prime}}-y_{\lambda^{\prime}}^{(l)}\right|<2^{-\left(\alpha_{n}+3 \varepsilon_{m}\right) j}\right\} \\
& \geq \#\left\{\lambda \in \Lambda_{j}: \sup _{\lambda^{\prime} \subset \lambda}\left|y_{\lambda^{\prime}}^{(l)}\right| \geq 2 \cdot 2^{-\left(\alpha_{n}+3 \varepsilon_{m}\right) j}\right\}-\#\left\{\lambda \in \Lambda_{j}: \sup _{\lambda^{\prime} \subset \lambda}\left|c_{\lambda^{\prime}}-y_{\lambda^{\prime}}^{(l)}\right| \geq 2^{-\left(\alpha_{n}+3 \varepsilon_{m}\right) j}\right\} \\
& \geq \#\left\{\lambda \in \Lambda_{j}: \sup _{\lambda^{\prime} \subset \lambda}\left|z_{\lambda^{\prime}}\right| \geq 2^{-\left(\alpha_{n}+2 \varepsilon_{m}\right) j}\right\}-\#\left\{\lambda \in \Lambda_{j}: \sup _{\lambda^{\prime} \subset \lambda}\left|c_{\lambda^{\prime}}-y_{\lambda^{\prime}}^{(l)}\right| \geq 2^{-\left(\alpha_{n}+3 \varepsilon_{m}\right) j}\right\}
\end{aligned}
$$




$$
\begin{aligned}
& \geq 2^{\left(\nu\left(\alpha_{n}\right)-\varepsilon_{m}\right) j}-2^{\left(\nu\left(\alpha_{n}\right)-2 \varepsilon_{m}\right) j} \\
& \geq 2^{\left(\nu\left(\alpha_{n}\right)-2 \varepsilon_{m}\right) j}
\end{aligned}
$$

using the choice of $j_{m, n, l}$. It follows that, for every $m, n \in \mathbb{N}$,

$$
\limsup _{j \rightarrow+\infty} \frac{\log \#\left\{\lambda \in \Lambda_{j}: \sup _{\lambda^{\prime} \subset \lambda}\left|c_{\lambda^{\prime}}\right| \geq 2^{-\left(\alpha_{n}+3 \varepsilon_{m}\right) j}\right\}}{\log 2^{j}} \geq \nu\left(\alpha_{n}\right)-2 \varepsilon_{m}
$$

Taking the limit as $m \rightarrow+\infty$, we get $\widetilde{\nu}_{\vec{c}}\left(\alpha_{n}\right) \geq \nu\left(\alpha_{n}\right)$ for every $n \in \mathbb{N}$. The conclusion follows from the right-continuity of the functions $\widetilde{\nu}_{\vec{c}}$ and $\nu$.

\section{Comparison with $\mathcal{S}^{\nu}$ spaces}

From the definition of the wavelet leaders, it is direct to see that $\nu_{\vec{c}} \leq \widetilde{\nu_{\vec{c}}}$ for any sequence $\vec{c} \in \Omega$ since $\left|c_{j, k}\right| \leq d_{j, k}$ for every $(j, k) \in \Lambda$. Therefore, given an admissible profile $\nu$, we have

$$
\mathcal{L}^{\nu} \subset \mathcal{S}^{\nu}
$$

We can also compare easily the topologies of $\mathcal{S}^{\nu}$ and $\mathcal{L}^{\nu}$. The proof is straightforward.

\section{Proposition 5.1.}

1. If $\alpha \in \mathbb{R}$ and $\beta \in\{-\infty\} \cup[0,+\infty[$, then we have

$$
\delta_{\alpha, \beta} \leq \widetilde{\delta}_{\alpha, \beta} \quad \text { and } \quad \widetilde{A}(\alpha, \beta) \subset A(\alpha, \beta) .
$$

2. If a sequence converges in $\left(\widetilde{A}(\alpha, \beta), \widetilde{\delta}_{\alpha, \beta}\right)$, it converges in $\left(A(\alpha, \beta), \delta_{\alpha, \beta}\right)$ to the same limit. If a sequence is a Cauchy sequence in $\left(\widetilde{A}(\alpha, \beta), \widetilde{\delta}_{\alpha, \beta}\right)$, it is a Cauchy sequence in $\left(A(\alpha, \beta), \delta_{\alpha, \beta}\right)$.

3. The space $\left(\widetilde{A}(\alpha, \beta), \widetilde{\delta}_{\alpha, \beta}\right)$ has a stronger topology than the topology induced by the distance $\delta_{\alpha, \beta}$.

4. The space $\left(\mathcal{L}^{\nu}, \widetilde{\delta}\right)$ has a stronger topology than the topology induced by the distance $\delta$.

The aim of this section is to study in which cases the space $\mathcal{L}^{\nu}$ gives more information than the space $\mathcal{S}^{\nu}$. More precisely, we will get a condition to have the strict inclusion of $\mathcal{L}^{\nu}$ into $\mathcal{S}^{\nu}$. Let us start with a case where the inclusion is always strict.

Proposition 5.2. If $\nu$ is an admissible profile such that $\alpha_{\min }=0$, then $\mathcal{L}^{\nu}$ is strictly included in $\mathcal{S}^{\nu}$.

Proof. Since $\mathcal{L}^{\nu}$ is always included in $C^{0}$, it suffices to find an element of $\mathcal{S}^{\nu}$ which does not belong to $C^{0}$. Such an element is given by the sequence $\vec{c} \in \Omega$ defined by $c_{j, 0}:=j$ and $c_{j, k}:=0$ for $k \neq 0$, at every scale $j \in \mathbb{N}_{0}$.

Let us now assume that $\alpha_{\min }>0$. Let us start by recalling the following result concerning the estimation of the spectrum of singularities of a given function (see [10]).

Proposition 5.3. Let $f$ be a locally bounded function and let us denote by $\vec{c}$ its coefficients in a given periodized wavelet basis of $L^{2}([0,1])$. If there is $r>0$ such that $\vec{c} \in C^{r}$, then for every $\alpha \geq 0$, we have

$$
d_{f}(\alpha) \leq \widetilde{\nu}_{\vec{c}}(\alpha) \leq \min \left\{\alpha \sup _{0<\alpha^{\prime} \leq \alpha} \frac{\nu_{\vec{c}}\left(\alpha^{\prime}\right)}{\alpha^{\prime}}, 1\right\}
$$


Let us also remark that $\widetilde{\nu}_{\vec{c}}(\alpha)=-\infty$ if and only if $\nu_{\vec{c}}(\alpha)=-\infty$.

Let $\nu$ be an admissible profile and let us set $h_{\max }:=\inf _{h \geq \alpha_{\min }} \frac{h}{\nu(h)}$. We define the function $\nu_{I}$ as follows:

$$
\nu_{I}(\alpha):= \begin{cases}-\infty & \text { if } \alpha<\alpha_{\min }, \\ \alpha \sup _{0<\alpha^{\prime} \leq \alpha} \frac{\nu\left(\alpha^{\prime}\right)}{\alpha^{\prime}} & \text { if } \alpha_{\min } \leq \alpha \leq h_{\max }, \\ 1 & \text { if } \alpha>h_{\max } .\end{cases}
$$

Of course, we have $\nu \leq \nu_{I}$. Since $\nu$ is right-continuous, we also get that $\nu_{I}$ is right-continuous. Therefore, $\nu_{I}$ is an admissible profile. Moreover, in [4], it is proved that there is $\vec{c} \in \mathcal{S}^{\nu}$ such that $\nu_{\vec{c}}=\nu$ on $\mathbb{R}$ and

$$
d_{f}(\alpha)= \begin{cases}\nu_{I}(\alpha) & \text { if } \alpha \leq h_{\max } \\ -\infty & \text { otherwise }\end{cases}
$$

where $f$ is the function whose coefficients in a given periodized wavelet basis of $L^{2}([0,1])$ are given by $\vec{c}$. Then, Proposition 5.3 directly implies what follows.

Proposition 5.4. If $\nu$ is an admissible profile such that $\alpha_{\min }>0$, there exists $\vec{c} \in \mathcal{S}^{\nu}$ such that $\nu_{\vec{c}}=\nu$ and $\widetilde{\nu}_{\vec{c}}=\nu_{I}$ on $\mathbb{R}$.

In fact, the consideration of $\nu_{I}$ instead of $\nu$ transforms the admissible profile into another admissible profile with an additional property, called the increasing-visibility (see [21]).

Definition 5.5. Take $0 \leq a<b \leq+\infty$. A function $g:[a, b] \mapsto[0,+\infty[$ is with increasing-visibility on $[a, b]$ if $g$ is continuous at $a$ and if the function

$$
x \mapsto \frac{g(x)}{x}
$$

is increasing on $] a, b]$.

In other words, a function $g$ is with increasing-visibility if for all $x \in] a, b]$, the segment $[(0,0),(x, g(x))]$ lies above the graph of $g$ on $] a, x]$. This property is exactly the necessary and sufficient condition on the admissible profile $\nu$ to have the equality of the spaces $\mathcal{L}^{\nu}$ and $\mathcal{S}^{\nu}$.

Theorem 5.6. Let $\nu$ be an admissible profile such that $\alpha_{\min }>0$. Then $\mathcal{L}^{\nu}=\mathcal{S}^{\nu}$ if and only if $\nu$ is with increasing-visibility on $\left[\alpha_{\min }, h_{\max }\right]$, i.e. if and only if $\nu=\nu_{I}$ on $\mathbb{R}$.

Proof. Let us first assume that $\mathcal{L}^{\nu}=\mathcal{S}^{\nu}$. From Proposition 5.4, we know that there is $\vec{c} \in \mathcal{S}^{\nu}$ such that $\nu_{\vec{c}}=\nu$ and $\widetilde{\nu}_{\vec{c}}=\nu_{I}$. Since $\vec{c} \in \mathcal{S}^{\nu}=\mathcal{L}^{\nu}$, we directly get that $\widetilde{\nu}_{\vec{c}} \leq \nu$, hence $\nu=\nu_{I}$.

Conversely, assume that $\nu=\nu_{I}$ on $\left[\alpha_{\min }, h_{\max }\right]$. If $\vec{c} \in \mathcal{S}^{\nu}$, then $\widetilde{\nu}_{\vec{c}} \leq \nu_{I}=\nu$ from Proposition 5.3 and it follows that $\vec{c} \in \mathcal{L}^{\nu}$.

Proposition 5.7. If $\mathcal{L}^{\nu}$ is strictly included in $\mathcal{S}^{\nu}$, then the set $\mathcal{L}^{\nu}$ is not closed in the space $\mathcal{S}^{\nu}$.

Proof. First let us remark that the sequences with only a finite number of non-zero coefficients belong to $\mathcal{L}^{\nu}$. Take now an element $\vec{c}$ of $\mathcal{S}^{\nu}$ which is not in $\mathcal{L}^{\nu}$. The "truncated" sequence $\left(\vec{c}^{N}\right)_{N \in \mathbb{N}}$ defined by

$$
c_{j, k}^{N}:= \begin{cases}c_{j, k} & \text { if } j \leq N \text { and } k \in\left\{0, \ldots, 2^{j}-1\right\} \\ 0 & \text { if } j>N \text { and } k \in\left\{0, \ldots, 2^{j}-1\right\}\end{cases}
$$


for all $N \in \mathbb{N}$, converges to $\vec{c}$ for the topology of $\mathcal{S}^{\nu}$ (see Lemma 6.3 in [5]) and each of its elements belongs to $\mathcal{L}^{\nu}$. Hence the conclusion.

Let us finish this section by looking for which admissible profile $\nu^{\prime}$ we have $\mathcal{S}^{\nu} \subset \mathcal{L}^{\nu^{\prime}}$.

Proposition 5.8. Let $\nu$ be an admissible profile such that $\alpha_{\min }>0$. Then $\mathcal{S}^{\nu} \subset \mathcal{L}^{\nu^{\prime}}$ if and only if $\nu^{\prime} \geq \nu_{I}$ on $\mathbb{R}$ and in this case, the inclusion map is continuous.

Proof. First, assume that $\mathcal{S}^{\nu} \subset \mathcal{L}^{\nu^{\prime}}$. Using Proposition 5.4, let $\vec{c} \in \mathcal{S}^{\nu}$ be such that $\nu_{\vec{c}}=\nu$ and $\widetilde{\nu}_{\vec{c}}=\nu_{I}$. Then $\vec{c} \in \mathcal{L}^{\nu^{\prime}}$ and it follows that $\nu_{I}=\widetilde{\nu}_{\vec{c}} \leq \nu^{\prime}$. Reciprocally, it suffices to show that $\mathcal{S}^{\nu} \subset \mathcal{L}^{\nu_{I}}$. If $\vec{c} \in \mathcal{S}^{\nu}$, we know from Proposition 5.3 that $\widetilde{\nu}_{\vec{c}} \leq \nu_{I}$. This means that $\vec{c} \in \mathcal{L}^{\nu_{I}}$.

Both $\mathcal{S}^{\nu}$ and $\mathcal{L}^{\nu^{\prime}}$ are complete metrizable topological vector spaces whose topologies are stronger than the pointwise convergence. The closed graph theorem gives the continuity.

Remark 5.9. If $\alpha_{\text {min }}=0$, the space $\mathcal{S}^{\nu}$ is not included in $\mathcal{L}^{\nu^{\prime}}$ for any admissible profile $\nu^{\prime}$ since it is not included in $C^{0}$.

\section{Comparison with Oscillation spaces}

In this last section, we investigate the relations existing between the $\mathcal{L}^{\nu}$ spaces and the Oscillation spaces. Let us recall the definition of the Oscillation spaces of sequences $\mathcal{O}_{p}^{s}$ (see [14]). There are the discrete counterparts of the Oscillation spaces of functions. These spaces are a particular case of Oscillation spaces $\mathcal{O}_{p}^{s, s^{\prime}}$ considered in $[15,11]$.

Definition 6.1. For $s \in \mathbb{R}$ and $p>0$, a sequence $\vec{c} \in \Omega$ belongs to $\mathcal{O}_{p}^{s}$ if

$$
\|\vec{c}\|_{\mathcal{O}_{p}^{s}}:=\sup _{j \in \mathbb{N}_{0}} 2^{\left(s-\frac{1}{p}\right) j}\left(\sum_{k=0}^{2^{j}-1} d_{j, k}^{p}\right)^{\frac{1}{p}}<+\infty .
$$

Let us remark that $\mathcal{O}_{p}^{s} \subset C^{0}$ so that if $\vec{c} \in \mathcal{O}_{p}^{s}$, then its wavelet leaders $d_{j, k}$ are finite. It is clear from the definition that $\mathcal{O}_{p}^{s} \subset b_{p, \infty}^{s}$ for every $s \in \mathbb{R}$ and $p>0$. Moreover, $\|\cdot\|_{\mathcal{O}_{p}^{s}}$ is a $(1 \wedge p)$-norm and so, $\left(\mathcal{O}_{p}^{s},\|\cdot\|_{\mathcal{O}_{p}^{s}}\right)$ is a complete topological vector space.

Let us note that a sequence $\vec{c}$ belongs to $\mathcal{L}^{\nu}\left(\right.$ resp. $\mathcal{O}_{p}^{s}$ ) if and only if the sequence defined by the wavelet leaders of $\vec{c}$ belongs to $\mathcal{S}^{\nu}$ (resp. $b_{p, \infty}^{s}$ ). Proposition 2.1 implies then directly the following embedding result.

Proposition 6.2. Let $\eta$ be the concave conjugate of $\nu$. For any dense sequence $\left(p_{n}\right)_{n \in \mathbb{N}}$ in $\mathbb{R}$ and for any sequence $\left(\varepsilon_{m}\right)_{m \in \mathbb{N}}$ of strictly positive numbers which decreases to 0 , we have

$$
\mathcal{L}^{\nu} \subset \bigcap_{p>0} \bigcap_{\varepsilon>0} \mathcal{O}_{p}^{\frac{\eta(p)}{p}-\varepsilon}=\bigcap_{n \in \mathbb{N}} \bigcap_{m \in \mathbb{N}} \mathcal{O}_{p_{n}}^{\frac{\eta\left(p_{n}\right)}{p_{n}}-\varepsilon_{m}} .
$$

Moreover, the inclusion becomes an equality if $\nu$ is concave.

Theorem 6.3. Let us assume that $\nu$ is concave and let $\eta$ be the concave conjugate of $\nu$. If $\left(p_{n}\right)_{n \in \mathbb{N}}$ is a dense sequence of $] 0,+\infty\left[\right.$ and if $\left(\varepsilon_{m}\right)_{m \in \mathbb{N}}$ is a sequence of strictly positive numbers converging to 0 , then

$$
\mathcal{L}^{\nu}=\bigcap_{p>0} \bigcap_{\varepsilon>0} \mathcal{O}_{p}^{\frac{\eta(p)}{p}-\varepsilon}=\bigcap_{n \in \mathbb{N}} \bigcap_{m \in \mathbb{N}} \mathcal{O}_{p_{n}}^{\frac{\eta\left(p_{n}\right)}{p_{n}}-\varepsilon_{m}},
$$


and the topology $\widetilde{\tau}$ on $\mathcal{L}^{\nu}$ defined as the weakest one such that each identity map $i:\left(\mathcal{L}^{\nu}, \widetilde{\tau}\right) \rightarrow \mathcal{O}_{p_{n}}^{\frac{\eta\left(p_{n}\right)}{p_{n}}-\varepsilon_{m}}$ is continuous, is equivalent to $\left(\mathcal{L}^{\nu}, \widetilde{\delta}\right)$.

Proof. The algebraic result is a consequence of Proposition 6.2. For every $n, m \in \mathbb{N}$, the topology of $\mathcal{O}_{p_{n}}^{\frac{\eta\left(p_{n}\right)}{p_{n}}-\varepsilon_{m}}$ is metrizable, complete and stronger than the topology of pointwise convergence. From Proposition 3.16, $\widetilde{\tau}$ is metrizable, complete and stronger than the topology of pointwise convergence. The closed graph theorem leads to conclusion.

Let us now show that, as in the case of $\mathcal{S}^{\nu}$, the concavity of $\nu$ is also a necessary condition to the equality between $\mathcal{L}^{\nu}$ and the intersection of Oscillation spaces. Let $\underline{\nu}$ be the concave hull of $\nu$ on $\left[\alpha_{\min },+\infty[\right.$, i.e. the smallest concave function $F$ on this interval which satisfies $F \geq \nu$ on this interval. This function is defined, continuous, non-decreasing on $\left[\alpha_{\min },+\infty[\right.$ and with values in $[0,1]$. Moreover, from Proposition 8.10 of [5], we know that

$$
\eta(p)=\inf _{\alpha \geq \alpha_{\min }}(1+p \alpha-\underline{\nu}(\alpha)), \quad p>0
$$

where $\eta$ is the concave conjugate of $\nu$. For $\alpha<\alpha_{\min }$, we set $\underline{\nu}(\alpha):=-\infty$.

Proposition 6.4. If $\underline{\nu}$ is the concave hull of $\nu$, we have

$$
\mathcal{L}^{\underline{\nu}}=\bigcap_{p>0} \bigcap_{\varepsilon>0} \mathcal{O}_{p}^{\frac{\eta(p)}{p}-\varepsilon} .
$$

Proof. This result follows from Proposition 6.2.

Proposition 6.5. If $\nu$ is not concave, then $\mathcal{L}^{\nu}$ is strictly included in $\mathcal{L}^{\underline{\nu}}$.

Proof. Using Proposition 4.1, let us consider $\vec{c} \in \mathcal{L}^{\underline{\nu}}$ such that $\widetilde{\nu}_{\vec{c}}=\underline{\nu}$. By assumption, there is $\alpha \geq \alpha_{\min }$ such that $\nu(\alpha)<\underline{\nu}(\alpha)=\widetilde{\nu}_{\vec{c}}(\alpha)$ and it follows that the sequence $\vec{c}$ does not belong to $\mathcal{L}^{\nu}$.

\section{Acknowledgments}

The authors would like to thank Stéphane Jaffard for the pertinent comments he made about our work which allowed to improve the quality of the manuscript. The second author was supported by a grant of Research Fellow from the Fonds National de la Recherche Scientifique (FNRS).

\section{References}

[1] J.M. Aubry, F. Bastin, Advanced topology on the multiscale sequence spaces $\mathcal{S}^{\nu}$, J. Math. Anal. Appl. 350 (2009) 439-454.

[2] J.M. Aubry, F. Bastin, Diametral dimension of some pseudoconvex multiscale spaces, Studia Math. 19 (1) (2010) $27-42$.

[3] J.M. Aubry, F. Bastin, A walk from multifractal analysis to functional analysis with $S^{\nu}$ spaces, and back, in: Recent Developments in Fractals and Related Fields, Springer, 2010, pp. 93-106.

[4] J.M. Aubry, F. Bastin, S. Dispa, Prevalence of multifractal functions in $S^{\nu}$ spaces, J. Fourier Anal. Appl. 13 (2) (2007) $175-185$

[5] J.M. Aubry, F. Bastin, S. Dispa, S. Jaffard, Topological properties of the sequence spaces $S^{\nu}$, J. Math. Anal. Appl. 321 (1) (2006) 364-387.

[6] J.M. Aubry, F. Bastin, S. Dispa, S. Jaffard, The spaces $S^{\nu}$ : new spaces defined with wavelet coefficients and related to multifractal analysis, Int. J. Appl. Math. Stat. 7 (2007) 82-95.

[7] J.M. Aubry, S. Jaffard, Random wavelet series, Comm. Math. Phys. 227 (2002) 483-514.

[8] F. Bastin, C. Esser, S. Jaffard, Large deviation spectra based on wavelet leaders, July 2014, submitted for publication.

[9] I. Daubechies, Ten Lectures on Wavelets, CBMS-NSF Regional Conf. Ser. in Appl. Math., 1992.

[10] C. Esser, T. Kleyntssens, S. Nicolay, A multifractal formalism for non concave and non increasing spectra: the $\mathcal{L}^{\nu}$ spaces approach, January 2015, submitted for publication. 
[11] S. Jaffard, Oscillation spaces: properties and applications to fractal and multifractal functions, J. Math. Phys. 39 (8) (1998) 4129-4141.

[12] S. Jaffard, On the Frisch-Parisi conjecture, J. Math. Pures Appl. 79 (6) (2000) 525-552.

[13] S. Jaffard, Beyond Besov spaces, Part 1: distributions of wavelet coefficients, J. Fourier Anal. Appl. 10 (2004) $221-246$.

[14] S. Jaffard, Wavelet techniques in multifractal analysis, fractal geometry and applications: a jubilee of Benoit Mandelbrot, Proc. Sympos. Pure Math. 72 (2004) 91-151.

[15] S. Jaffard, Beyond Besov spaces, Part 2: oscillation spaces, Constr. Approx. 21 (2005) 29-61.

[16] S. Jaffard, B. Lashermes, P. Abry, Wavelet leaders in multifractal analysis, in: Wavelet Analysis and Applications, in: Appl. Numer. Harmon. Anal., 2006, pp. 201-246.

[17] H. Jarchow, Locally Convex Spaces, Teubner, Stuttgart, 1981.

[18] T. Kleyntssens, C. Esser, S. Nicolay, A multifractal formalism based on the $\mathcal{S}^{\nu}$ spaces: from theory to practice, November 2014, submitted for publication.

[19] P.G. Lemarié, Y. Meyer, Ondelettes et bases hilbertiennes, Rev. Math. Iberoam. 1 (1986) 1-18.

[20] S. Mallat, A Wavelet Tour of Signal Processing, Academic Press, 1999.

[21] D. Maman, S. Seuret, Fixed points for the multifractal spectrum application, submitted for publication.

[22] Y. Meyer, Ondelettes et opérateurs, Hermann, 1990.

[23] G. Parisi, U. Frisch, On the singularity structure of fully developed turbulence, in: Turbulence and Predictability in Geophysical Fluid Dynamics, 1985, pp. 84-87. 